ARTICLE

\title{
Nuclear condensates of p300 formed though the structured catalytic core can act as a storage pool of p300 with reduced HAT activity
}

Yi Zhang (1) ${ }^{1}$, Kyle Brown², Yucong Yu ${ }^{3}$, Ziad Ibrahim4, Mohamad Zandian (1) 1, Hongwen Xuan ${ }^{3}$, Steven Ingersoll ${ }^{2}$, Thomas Lee ${ }^{5}$, Christopher C. Ebmeier (D) ${ }^{5}$, Jiuyang Liu', Daniel Panne ${ }^{4}$, Xiaobing Shi(D) ${ }^{3}$, Xiaojun $\operatorname{Ren}^{2} \&$ Tatiana G. Kutateladze (1) ${ }^{1 凶}$

The transcriptional co-activator and acetyltransferase p300 is required for fundamental cellular processes, including differentiation and growth. Here, we report that p300 forms phase separated condensates in the cell nucleus. The phase separation ability of p300 is regulated by autoacetylation and relies on its catalytic core components, including the histone acetyltransferase (HAT) domain, the autoinhibition loop, and bromodomain. p300 condensates sequester chromatin components, such as histone $\mathrm{H} 3$ tail and DNA, and are amplified through binding of p300 to the nucleosome. The catalytic HAT activity of p300 is decreased due to occlusion of the active site in the phase separated droplets, a large portion of which co-localizes with chromatin regions enriched in H3K27me3. Our findings suggest a model in which p300 condensates can act as a storage pool of the protein with reduced HAT activity, allowing p300 to be compartmentalized and concentrated at poised or repressed chromatin regions.

\footnotetext{
${ }^{1}$ Department of Pharmacology, University of Colorado School of Medicine, Aurora, CO, USA. ${ }^{2}$ Department of Chemistry, University of Colorado, Denver, CO, USA. ${ }^{3}$ Center for Epigenetics, Van Andel Research Institute, Grand Rapids, MI, USA. ${ }^{4}$ Department of Molecular and Cell Biology, Leicester Institute of Structural and Chemical Biology, University of Leicester, Leicester, UK. ${ }^{5}$ Department of Biochemistry, University of Colorado, Boulder, CO, USA.

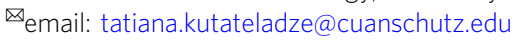


F ormation of condensates in the cell nucleus has been shown to drive the assembly of nuclear bodies, Cajal bodies, nucleoli, and speckles ${ }^{1,2}$. These membraneless compartments are produced via the liquid-liquid phase separation (LLPS) mechanisms and depend on weak, multivalent interactions involving intrinsically disordered regions (IDRs) of biomolecules $^{3-5}$. Several nuclear proteins and chromatin regulators, including HP1, BRD4, CBX, and MORC3, have been shown to form condensates with their phase separation ability being necessary for biological functions and subnuclear localization of these proteins ${ }^{6-16}$.

The transcriptional co-activator p300 is an acetyltransferase that is frequently dysregulated in disease, particularly cancer. p300 associates with over 400 binding partners, including transcription factors, DNA-binding proteins and subunits of the RNA polymerase II complex, to form activation complexes and facilitate transcription ${ }^{17-21}$. p300 acetylates histones and a number of essential nonhistone proteins, notably p53, E2F1, and androgen and estrogen receptors ${ }^{22-24}$. The highly selective acetylation of the H3K27 and H3K18 sites by $\mathrm{p} 300$ requires a cooperative action of its two functional domains: acetyllysine-binding bromodomain (BD) and the $\mathrm{H} 3$ tail-binding $\mathrm{ZZ}$ domain ${ }^{25}$.

p300 and its close homolog CBP share the same domain architecture, consisting of several conserved modules, including the catalytic histone acetyltransferase (HAT) domain. Located in the middle of the protein, the HAT domain is surrounded by $\mathrm{BD}$, a RING finger, and a plant homeodomain finger (PHD) from one side and the $\mathrm{ZZ}$ domain from another side that together comprise the catalytic core of p300 (Fig. 1a). The p300 catalytic activity is regulated through acetylation/deacetylation of a long loop in the HAT domain, termed the autoinhibitory loop (AIL), which in a hypoacetylated form impedes the acetyltransferase activity of p300 but releases the inhibition upon hyper autoacetylation ${ }^{17,26}$. Structural studies of the p300 region encompassing BD, RING, PHD and the HAT domain suggest an additional layer of p300 regulation through the RING finger that contributes to the inhibition by occluding the HAT active site ${ }^{27}$. Furthermore, the BD-RING-PHD-HAT region of p300 has been shown to form a dimer, in which the hypoacetylated form of AIL of one molecule inserts in the active site of the HAT domain from another molecule and thus undergoes 'in trans' autoacetylation, a reaction that is controlled by signal-dependent transcription factor dimerization $^{28}$. Genome-wide-mapping analysis reveals that p300/CBP generally occupies transcriptionally active chromatin marked by the active H3K27ac modification, however a large number of silent or poised genes marked by the repressive H3K27me3 modification and bound by p300/CBP has also been identified $^{25,29-33}$. In cells, p300/CBP is ubiquitously expressed and can form discrete foci in the nucleus and localize to PML nuclear bodies ${ }^{34-37}$.

In this study, we show that the structured catalytic core of p300 undergoes phase separation and forms liquid condensates in cells and in vitro. The acetylation state of p300 modulates its ability to phase separate through attenuating intermolecular interactions involving the autoinhibition loop, the HAT domain, and BD. Our data suggest a model for compartmentalization and concentration of a pool of p300 with reduced catalytic activity at poised or repressed chromatin regions through the formation of phaseseparated condensates.

\section{Results and discussion}

p300 forms dynamic condensates in cells. p300 is highly abundant in the cell nucleus and can shuttle between the nucleoplasmic and cytoplasmic fractions ${ }^{38-40}$. To characterize the distribution of p300, we transfected HeLa cells with YFP-tagged full-length p300 (YFP-p300 $\mathrm{FL}$ ) and monitored the localization of YFP-p300 $\mathrm{FL}$ in live cells by fluorescence microscopy (Fig. 1b-d). In the majority of cells assayed, YFP-p300 ${ }_{\mathrm{FL}}$ was somewhat diffusely distributed throughout the nucleus, but $15-30 \%$ of cells displayed nuclear speckles various in size (Fig. 1b). These data indicate the presence of discrete YFP-p $300_{\mathrm{FL}}$ compartments with elevated protein concentration and are in line with previous reports showing that both endogenous and expressed p300 (and its homolog CBP) form dynamic nuclear bodies ${ }^{34-37}$.

To determine whether p300 is mobile in the speckles, we measured the diffusion kinetics of selected p300 puncta by fluorescence recovery after photobleaching (FRAP) experiments. For each selected region, the laser beam was applied after two initial scans, and cell images were collected at $10 \mathrm{~s}$ intervals for a duration of $280 \mathrm{~s}$ (Fig. 1c, d). The experiments were performed on seven cells, and changes in the fluorescence signal of each bleached region were analyzed. The averaged signal intensity, which was normalized and plotted (Fig. 1c), showed a $\sim 60 \%$ recovery of the bleached regions' fluorescence with a half-life $\left(t_{1 / 2}\right)$ of $59 \mathrm{~s}$. Because the photobleaching irreversibly abolishes fluorescence, the recovery of the signal implies that YFP-p $300_{\mathrm{FL}}$ readily moves from unbleached regions to the bleached region. The fast signal recovery, and therefore the fast diffusion of $\mathrm{p} 300$, suggest that YFP-p $300_{\mathrm{FL}}$ speckles are viscous liquid droplets characterized by rapid protein exchange with the surrounding environment. Collectively, these results demonstrate that full length p300 forms dynamic nuclear condensates with liquid/gel-like properties.

The catalytic core of p300 phase separates into liquid droplets in cells and in vitro. The middle part of $\mathrm{p} 300$, consisting of the BD followed by the RING, PHD, HAT, and ZZ domains, comprises the catalytic core that we postulated might be involved in the phase separation process. To test this idea, we generated YFPtagged truncated p300 construct (aa 1024-1830 of p300), which in addition to the catalytic core contains a TAZ2 domain and is referred to as $\mathrm{p} 300_{\mathrm{BRPHZT}}$. We have previously shown that

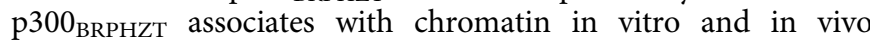
comparably to full-length $\mathrm{p} 300^{25}$. The YFP-p300 BRPHZT $_{\text {protein }}$ was produced using a doxycycline (DOX) inducible pTripZ vector, and expression of $\mathrm{p} 300_{\mathrm{BRPHZT}}$ was visualized in live HeLa cells. Much like the full-length protein, YFP-p300 $0_{\text {BRPHZT }}$ localized primarily to the nucleus and formed foci of various sizes (Supplementary Fig. 1). Furthermore, FRAP experiments showed a $\sim 80 \%$ fluorescence recovery with $t_{1 / 2}=29 \mathrm{~s}$, indicating that YFPp300 ${ }_{\text {BRPHZT }}$ and full length YFP-p300 have similar capabilities to form dynamic condensates in cells (Fig. 1e).

The cell nucleus is a highly crowded and viscous compartment packed with DNA, histones, and a multitude of nuclear proteins. To mimic molecular crowding, polyethylene glycol (PEG) is frequently used in vitro. Addition of PEG (PEG3350) to a solution containing the purified catalytic core, p300 ${ }_{\mathrm{BRPHZ}}$ (aa $1035-1720$ of p300), rapidly induced cloudiness in the originally clear solution, indicative of the conversion to a heterogenous suspension (described in detail below). Under a microscope, we observed spherical droplets of various sizes, suggesting the formation of liquid-liquid phase-separated condensates of the catalytic core of p300 (Fig. 1f). About $35 \%$ of p $300_{\mathrm{BRPHZ}}$ remained in the supernatant after removing the droplets by centrifugation (Supplementary Fig. 2).

Self-acetylation of p300 impairs its ability to phase separate. We found that different batches of purified p $300_{\text {BRPHZ }}$ showed variable ability to form droplets under otherwise identical conditions (Figs. If and 2a, b). Recombinantly expressed p300 is auto-acetylated to varying degrees ${ }^{26,41}$. We, therefore, assessed 
a

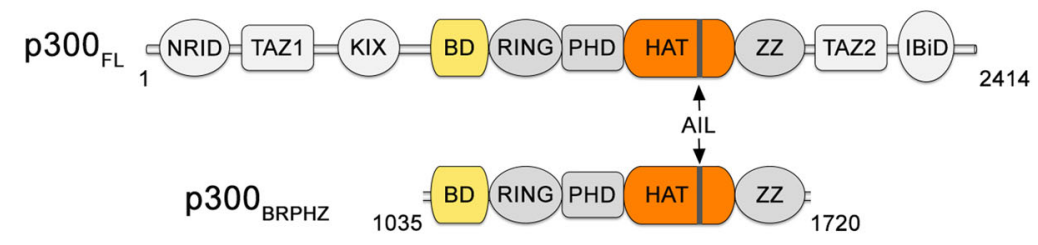

$b$
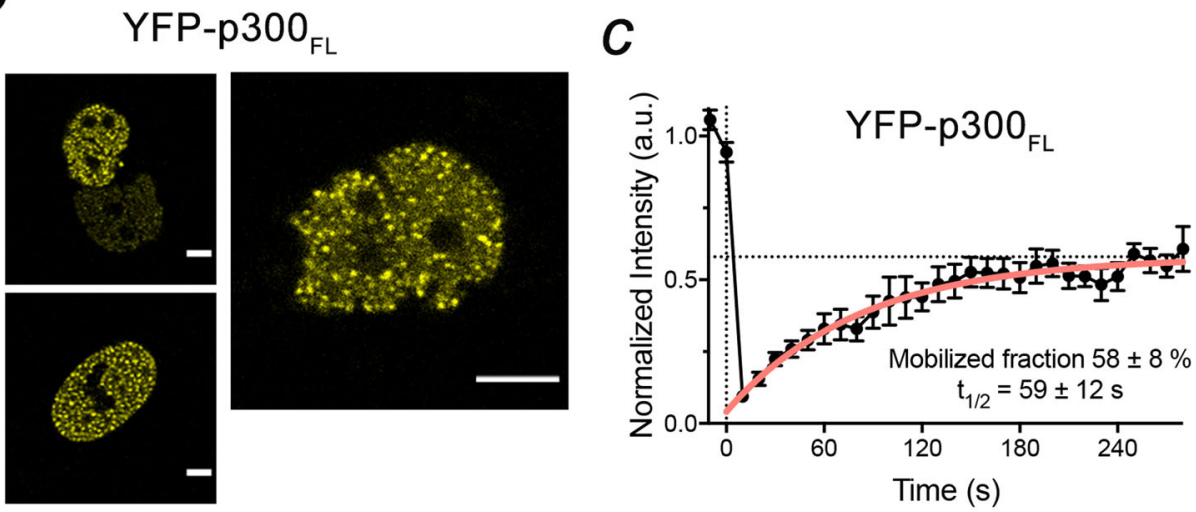

\section{$d_{\text {YFP-p300 }}$}

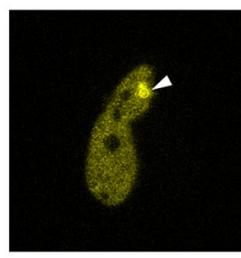

pre-bleach

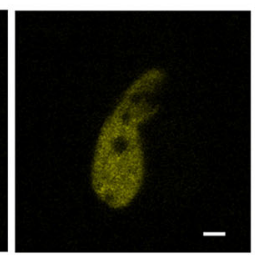

after-bleach

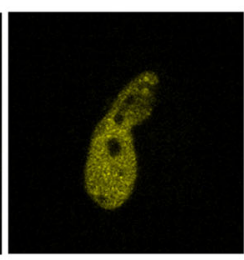

$30 \mathrm{~s}$

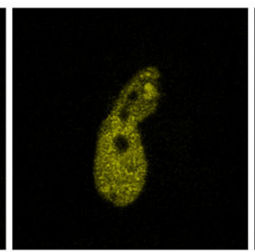

$150 \mathrm{~s}$

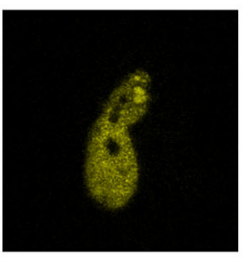

$280 \mathrm{~s}$
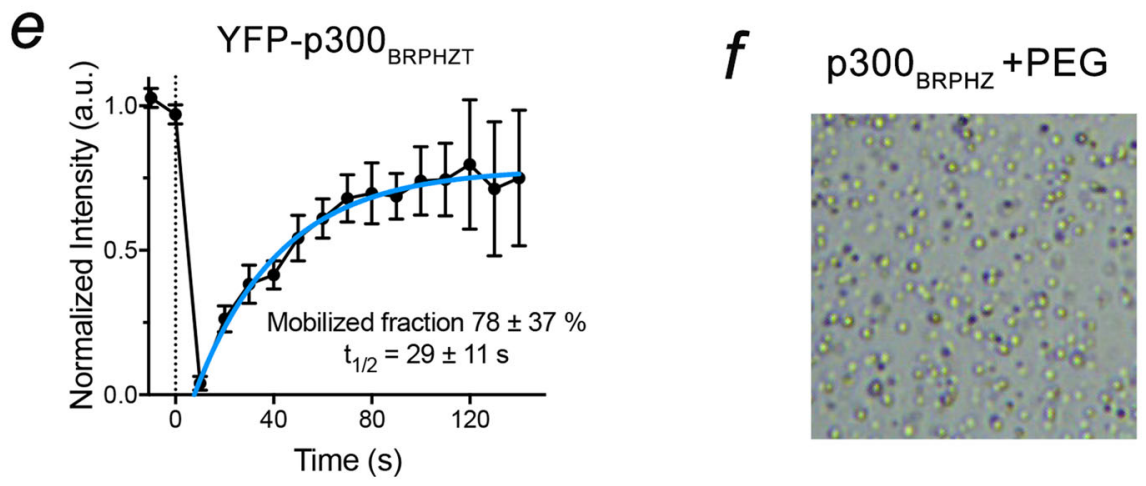

Fig. 1 p300 phase separates to condensates in living cells. a Schematic of $p 300_{\mathrm{FL}}$ and $\mathrm{p} 300_{\mathrm{BRPHZ}}$. b Representative images of HeLa cells expressing YFP$\mathrm{p} 300_{\mathrm{FL}}$. Scale bar, $5 \mu \mathrm{m}$. c FRAP curve of YFP-p300 FL was obtained from averaging data from six HeLa cells. Error bars represent SEM. $n=6$ cells examined over three independent experiments. d Representative FRAP images of YFP-p300 FL expressed in HeLa cells. The images were taken before and after photobleaching at indicated time points. The bleached condensate is indicated by a white arrowhead. Scale bar, $5 \mu$ m. e FRAP curve of YFP-p300 ${ }_{\text {BRPHZT }}$ condensates in HeLa cells. The FRAP curve was obtained from averaging data from four cells. Error bars represent SEM. $\mathbf{f} A$ representative image of a

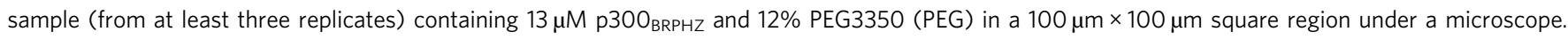

the self-acetylation levels of two different batches of purified p $300_{\text {BRPHZ }}$ by liquid chromatography-mass spectrometry (LC-MS). Acetylation levels of $\mathrm{p} 300_{\mathrm{BRPHZ}}$ were highly heterogeneous, containing a series of species with discrete molecular mass. For example, purification 1 contained $\mathrm{p} 300_{\mathrm{BRPHZ}}$ with molecular mass ranging from $\sim 81$ to $81.7 \mathrm{kDa}$ (Fig. $2 \mathrm{a}, \mathrm{c}$, and d). The increment of each mass peak was $\sim 42 \mathrm{Da}$, the mass of one acetyl group. Compared with its theoretical mass of $80,548.5 \mathrm{Da}$, recombinantly expressed $\mathrm{p} 300_{\mathrm{BRPHZ}}$ from purification 1 was acetylated on 13-25 lysine residues, with a median of 16 acetylated lysines (Fig. 2c, d). The sequence of p300 $0_{\mathrm{BRPHZ}}$ contains 60 lysine residues, indicating that $\sim 20-40 \%$ of lysine residues were acetylated. The $\mathrm{p} 300_{\mathrm{BRPHZ}}$ protein from purification 2 had on average 26 acetylated lysines. p300 ${ }_{\mathrm{BRPHZ}}$ with the lower 


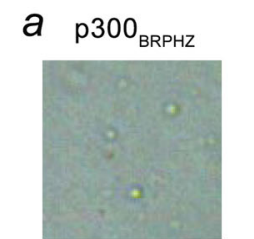

purification 1

$\sim 16 \mathrm{Kac}$

d

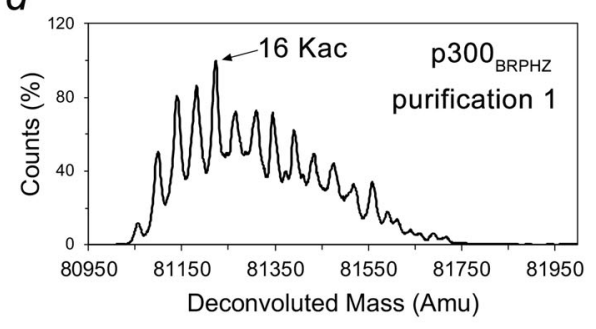

e

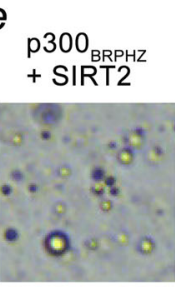

purification 1

$\sim 4 \mathrm{Kac}$

$h$

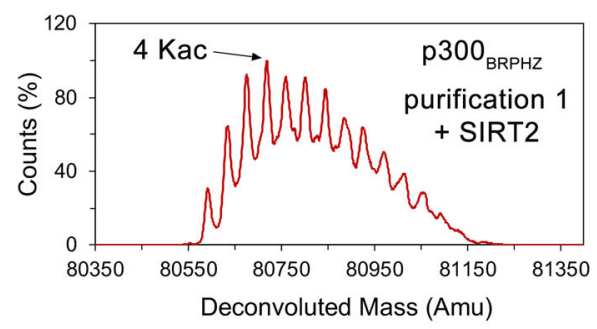

C

p300 ${ }_{\text {BRPHZ }}$ purification 1

\begin{tabular}{ccc}
\hline Mass & Acetyl-groups & theoretical Mass \\
\hline 81099 & 13 & 81099 \\
81139 & 14 & 81139 \\
81181 & 15 & 81181 \\
81222 & 16 & 81222 \\
81266 & 17 & 81266 \\
81309 & 18 & 81309 \\
81345 & 19 & 81345 \\
81390 & 20 & 81390 \\
81432 & 21 & 81432 \\
81475 & 22 & 81475 \\
81519 & 23 & 81519 \\
81557 & 24 & 81557 \\
\hline
\end{tabular}

$g$

p300 ${ }_{\text {BRPHZ }}$ purification $1+$ SIRT2

\begin{tabular}{ccc}
\hline Mass & Acetyl-groups & theoretical Mass \\
\hline 80591 & 1 & 80591 \\
80634 & 2 & 80634 \\
80675 & 3 & 80675 \\
80718 & 4 & 80719 \\
80759 & 5 & 80759 \\
80801 & 6 & 80802 \\
80845 & 7 & 80845 \\
80885 & 8 & 80885 \\
80924 & 9 & 80924 \\
80969 & 10 & 80969 \\
81011 & 11 & 81011 \\
81051 & 12 & 81051 \\
81091 & 13 & 81091 \\
\hline
\end{tabular}

i

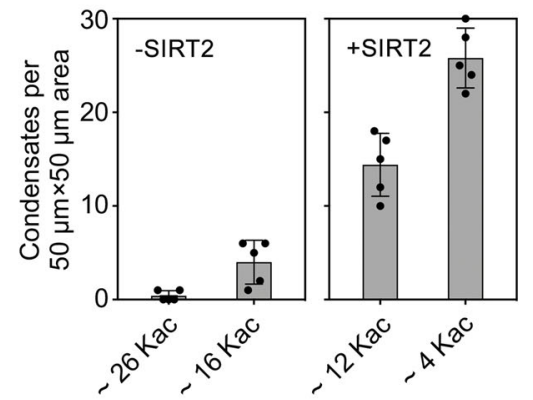

$j$

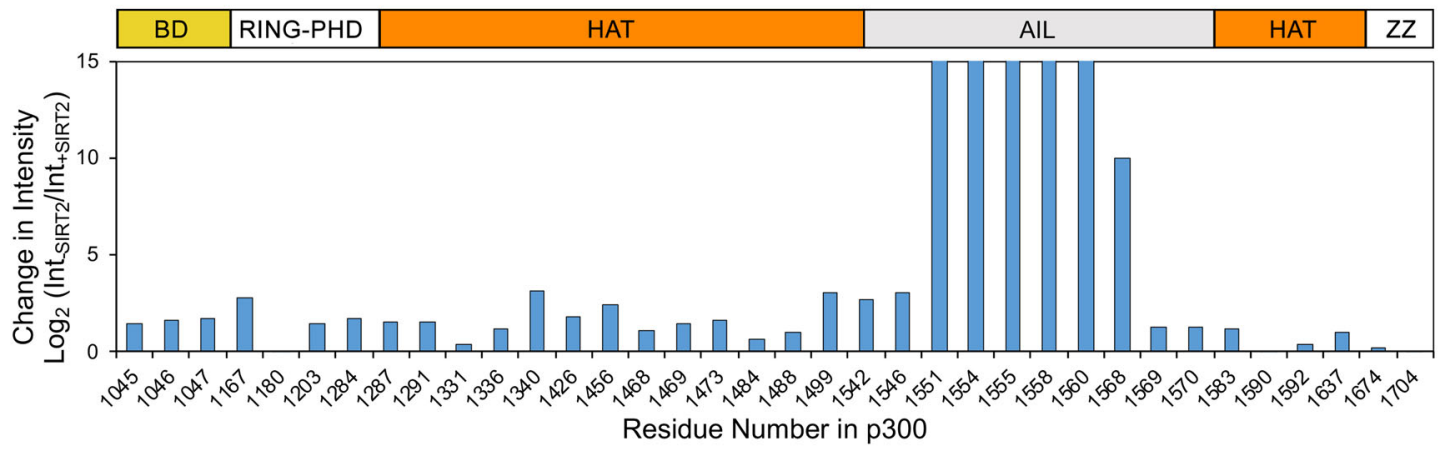

acetylation level (purification 1) visibly formed condensates in the presence of PEG (Fig. 2a), while p300 $0_{\text {BRPHZ }}$ with the higher acetylation level (purification 2) showed almost no droplet formation (Fig. 2b). Together, these results suggest a negative correlation between the acetylation level of p300 and its ability to form condensates in vitro.
We further examined whether acetylation levels modulate the condensation behavior of $\mathrm{p} 300_{\mathrm{BRPHZ}}$. The $\mathrm{NAD}^{+}$-dependent histone deacetylase SIRT2 has been shown to selectively and uniquely deacetylate p300 in cells and in vitro ${ }^{42}$. We incubated p300 $0_{\text {BRPHZ }}$ with SIRT2 and $\mathrm{NAD}^{+}$and then purified the protein by size-exclusion chromatography. This reduced the number of 
Fig. 2 p300 $_{\mathrm{BRPHZ}}$ is heterogeneously acetylated and SIRT2-induced deacetylation promotes phase separation of p300 ${ }_{\mathrm{BRPHZ}} \cdot \mathbf{a}$, b Representative images of two independently expressed and purified p $300_{\mathrm{BRPHZ}}$ samples containing $13 \mu \mathrm{M}$ protein and $12 \%$ PEG in a $50 \mu \mathrm{m} \times 50 \mu \mathrm{m}$ square region on cover slides under a microscope. c, d Liquid chromatography-mass spectrometry (LC-MS) analysis of $\mathrm{p}^{300_{\mathrm{BRPHZ}}}$ shown in (a). A table of peak mass and the corresponding number of acetylated lysine residues identified by mass spectrometry analysis are shown in (c) and (d). e, $\mathbf{f}$ Representative images of p300 $0_{\text {BRPHZ }}$ samples shown in (a) and (b) were treated with the deacetylase SIRT2. $\mathbf{g}, \mathbf{h}$ LC-MS analysis of p300 ${ }_{\text {BRPHz }}$ shown in (e). A table of peak mass and the corresponding number of acetylated lysine residues identified by mass spectrometry analysis are shown in ( $\mathbf{g})$ and (h). i Quantification of droplets counted in images acquired for the samples in ( $\mathbf{a}, \mathbf{b}, \mathbf{e}, \mathbf{f})$. The number of droplets was counted in five non-overlapping $50 \mu \mathrm{m} \times 50 \mu \mathrm{m}$ square regions and the mean value was plotted. Error bars represent SD. $\mathbf{j}$ Acetylated sites in $\mathrm{p} 300_{\mathrm{BRPHZ}}$ identified by LC-MS/MS. Vertical bars indicate differences in signal intensities of identical tryptic peptides derived from untreated vs. SIRT2-treated samples on the $\log _{2} \mathrm{scale}$. The highest intensities exceeding the plot were essentially qualitative, as they were only present in the untreated sample. A schematic representation of $\mathrm{p} \mathrm{BO}_{\mathrm{BRPHZ}}$ is shown above. Source data are provided in the Source Data file.

acetylated lysines in $\mathrm{p} 300_{\mathrm{BRPHZ}}$ (purification 1) from a median of 16 to a median of 4 (Fig. $2 \mathrm{c}, \mathrm{d}, \mathrm{g}, \mathrm{h}$ ). The same treatment of p300 ${ }_{\text {BRPHZ }}$ (purification 2) reduced the acetylation level from a median of 26 to 12 acetylated lysines. Generally, lower acetylation levels were correlated with an increased propensity to form droplets (Fig. 2i).

To identify regions in p300 affected by SIRT2 treatment, we analyzed tryptic peptides derived from untreated and SIRT2treated $\mathrm{p} 300_{\mathrm{BRPHZ}}$ using liquid chromatography-tandem mass spectrometry (LC-MS/MS). 49 out of 60 lysine residues were acetylated in untreated p300 ${ }_{\text {BRPHZ }}$. Comparison of the signal intensities of peptides revealed substantial differences in acetyla-

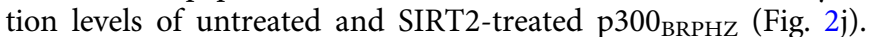
The most notable changes were observed for K1551, K1554, $\mathrm{K} 1555$, K1557, and K1560 located in the AIL, as acetylation of these lysine residues was not detectable in the SIRT2-treated

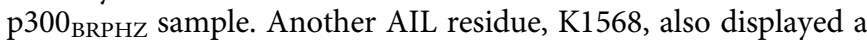
considerable reduction in signal intensity upon SIRT2 treatment. In contrast, signal intensity for lysine residues located in other regions of $\mathrm{p} 300_{\mathrm{BRPHZ}}$ was reduced to a substantially lesser degree. These data corroborate the idea that p300 self-acetylates a wide range of lysine residues spanning the catalytic BD-RING-PHD-HAT-ZZ core, and that the auto-inhibitory loop undergoes fast deacetylation, whereas deacetylation of other regions of $\mathrm{p} 300_{\mathrm{BRPHZ}}$ occurs much slower.

Because our data suggest that acetylation of p300 negatively regulates its ability to phase separate, we reasoned that acetylation should decrease or even eliminate the PEG-induced p $300_{\text {BRPHZ }}$ droplet formation. To test this, we first measured the HAT activity of untreated and SIRT2-treated p300 ${ }_{\mathrm{BRPHZ}}$ in the presence of acetyl-CoA by monitoring the release of the CoA product over time. As expected, SIRT2-treated p300 $0_{\text {BRPHZ }}$ produced more CoA than untreated $\mathrm{p} 300_{\mathrm{BRPHZ}}$, due to the higher number of unmodified lysine residues (substrates) present in SIRT2-treated p300 ${ }_{\text {BRPHZ }}$ (Fig. 3a). The acetylation reaction was fast and completed before $20 \mathrm{~min}$, in keeping with the previously reported activity of the p300 HAT domain ${ }^{41}$. Adding acetyl-CoA to a SIRT2-treated $\mathrm{p} 300_{\mathrm{BRPHZ}} / \mathrm{PEG}$ suspension led to a decrease in the cloudiness of the sample within 1 min (Fig. 3b), and liquid droplets were no longer visible under the microscope (Fig. 3c, d, and Supplementary Fig. 3a), indicating a disruption of LLPS due to the HAT reaction. Collectively, these data suggest that auto-acetylation of the AIL decreases the formation of p300 ${ }_{\text {BRPHZ }}$ condensates.

Phase separation of p300 relies on both BD and AIL. Weak and often nonspecific multivalent interactions are believed to drive phase separation ${ }^{1,4,7,8}$. The p300 HAT domain has been reported to associate with the hypoacetylated AIL from another p300 molecule 28 and BD of CBP was shown to bind the AIL peptide acetylated at $\mathrm{K} 1596^{43}$. Accordingly, we envisage two distinct mechanisms for the transition of the hyperacetylated p300 catalytic core (incapable of phase separation) to the hypoacetylated p300 catalytic core (capable of phase separation), which rely on intermolecular 'in trans' HAT-AIL or BD-acetyl-lysine (Kac) interactions (Fig. 3e). To test these possibilities, we examined the role of the BD and AIL in the formation of condensates. We used a deletion of the entire autoinhibitory loop $(\triangle \mathrm{AIL})$ in $\mathrm{p} 300_{\mathrm{BRPHZ}}$ or a mutation N1132A, which was previously shown to abrogate acetyllysine binding of the $\mathrm{BD}^{27}$.

Compared to the WT $\mathrm{p} 300_{\mathrm{BRPHZ}}$ protein that contained on average $\sim 12 \mathrm{Kac}$ sites and readily underwent phase separation (Fig. 3f), the N1132A mutant did not form PEG-induced droplets, despite having a similar acetylation level ( $\sim 14$ Kac sites)

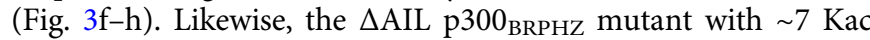
sites formed less visible condensates than the WT protein with $\sim 4$ Kac sites (Fig. 3i-k). We note that although the entire AIL was deleted, the $\triangle$ AIL $\mathrm{p} 300_{\mathrm{BRPHZ}}$ mutant was still acetylated at 7 lysine residues located outside this loop in the BD-RING-PHD-HAT-ZZ region. These data suggest that both HAT-AIL and BD-Kac-binding mechanisms contribute to p300 $0_{\text {BRPHZ }}$ phase separation. In a hyper-acetylated form where both the AIL and other regions of $\mathrm{p} 300_{\mathrm{BRPHZ}}$ are acetylated, $\mathrm{BD}$ may favor intramolecular 'in cis' contacts with the acetylated AIL, limiting the probability of intermolecular interactions and leading to a diffused distribution of $\mathrm{p} 300_{\mathrm{BRPHZ}}$. Upon SIRT2-treatment of WT $\mathrm{p} 300_{\mathrm{BRPHZ}}$, deacetylation of the AIL results in the release of $\mathrm{BD}$, allowing intermolecular 'in trans' $\mathrm{BD}-\mathrm{Kac}$ interactions and both intra- and intermolecular HAT-AIL interactions (Fig. 3e).

In support of this model, SIRT2 treatment of N1132A p300 $0_{\text {BRPHZ }}$, which did not originally phase separate (Fig. $4 \mathrm{a}$, left panel), led to the condensate formation (Fig. 4a, right panel, and Fig. 4b), suggesting that deacetylation of AIL promotes phase separation through the HAT-AIL interaction (Fig. 4c). Furthermore, both SIRT2-treated or untreated $\triangle \mathrm{AIL}$ p300 $\mathrm{BRPHZ}_{\mathrm{BH}}$ formed droplets, although to a lesser degree compared to the WT protein, reinforcing the role of the $\mathrm{BD}-\mathrm{Kac}$ interaction in promoting phase separation (Fig. 4d-f). Moreover, the catalytically impaired mutant D1399A p300 ${ }_{\mathrm{BRPHZ}}$ showed the formation of the droplets through the HAT-AIL interaction, since no self-acetylation occurred in this mutant (Fig. 4g, h, and Supplementary Fig. 2b, c). The SIRT2-dependent phase separation was also observed in the $\mathrm{p} 300_{\mathrm{HZ}}$ construct containing only the HAT and $\mathrm{ZZ}$ domains (Fig. 4i, j), whereas further deletion of AIL completely abolished droplet formation, regardless of the SIRT2 treatment (Fig. 4k, l). Lastly, both N1132A and $\triangle$ AIL mutants of YFP-p300 ${ }_{\mathrm{FL}}$ formed droplets in HeLa cells, confirming that full-length p300 can phase separate through either HAT-AIL interaction in N1132A YFP-p $300_{\mathrm{FL}}$ or $\mathrm{BD}$-acetyl-lysine interaction in $\triangle \mathrm{AIL}$ YFP-p300 FL (Fig. 4m).

To further confirm our model, we performed small-angle X-ray scattering (SAXS) experiments on purified WT p300 $340-2094$ and $\triangle$ AIL p300 $340-2094$ proteins at various time points before and after incubating with acetyl-CoA (Fig. 5a). SAXS experiments provide 
a

P300 $0_{\text {BRPHZ }}$

- - SIRT2 $=+$ SIRT2

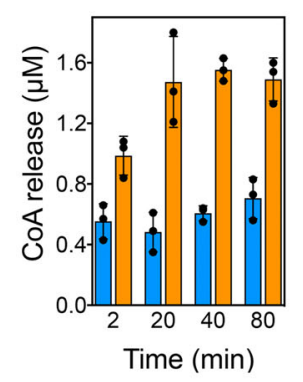

$d$

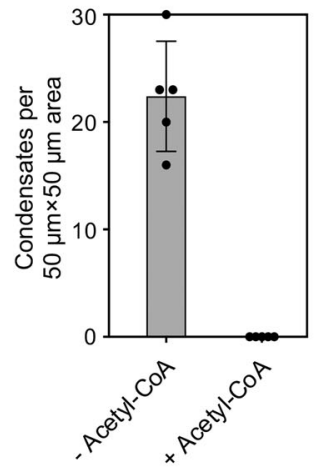

$b$

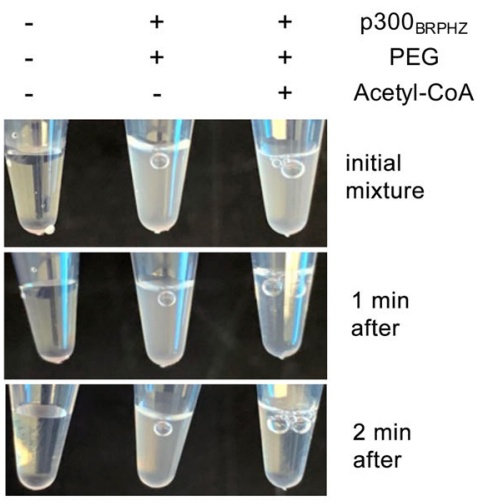

C

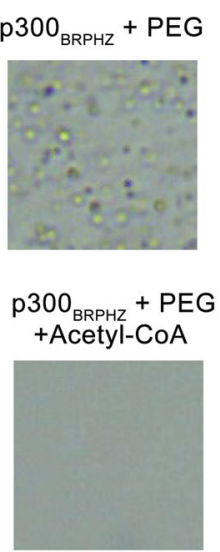

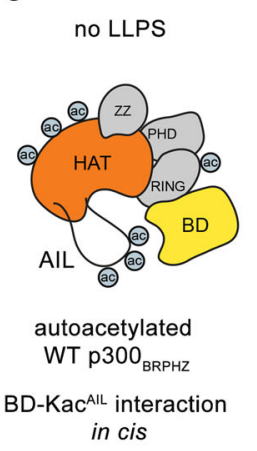

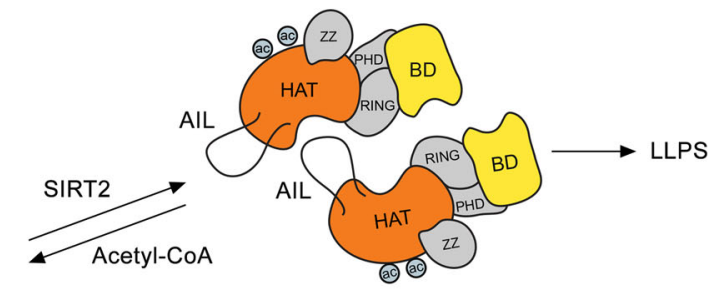

AIL-HAT interaction in trans
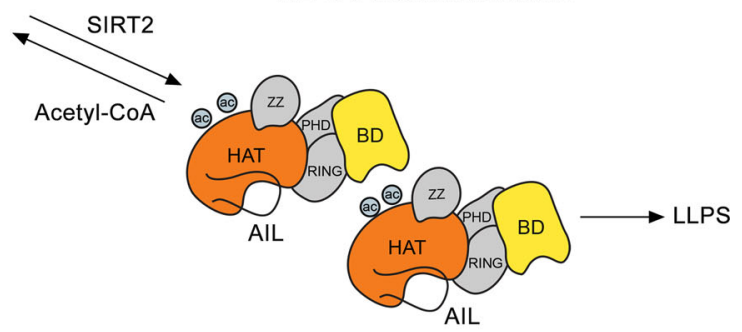

BD-Kac interaction in trans

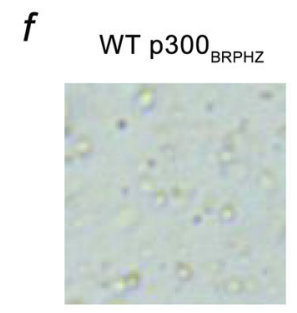

$\sim 12 \mathrm{Kac}$

i

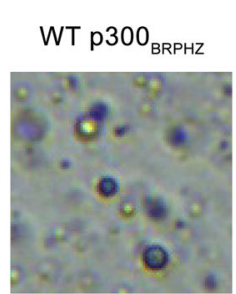

$\sim 4 \mathrm{Kac}$ $g$

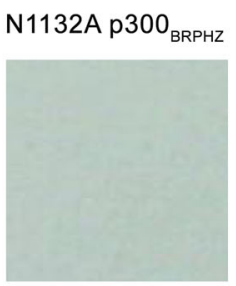

$\sim 14 \mathrm{Kac}$

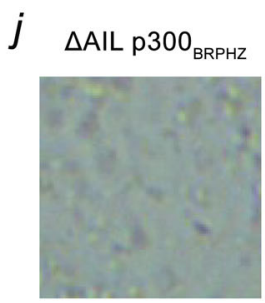

$\sim 7 \mathrm{Kac}$

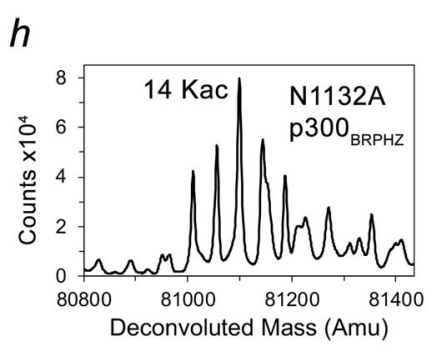

$k$

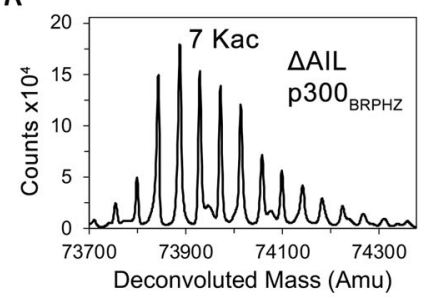

information about the protein's size and shape in solution. For WT p300 ${ }_{340-2094}$, autoacetylation led to little changes in the SAXS profile: the $\mathrm{Rg}$ value remained unchanged at $\sim 10 \mathrm{~nm}$, in agreement with monomeric $\mathrm{p} 300$, possibly because the acetylated AIL engages the BD 'in cis' (Fig. 5b, top panel). In contrast, for $\triangle$ AIL p300 $340-2094$, the Rg value increased over time (to $\sim 35 \mathrm{~nm}$ ) after autoacetylation for $1.5 \mathrm{~h}$ resulting in spherical droplets with a maximum dimension of $\sim 125 \mathrm{~nm}$ (Fig. 5a, bottom panel). These droplets likely arise through 'in trans' engagement of the BD with acetylated lysine residues outside AIL (Fig. 5b, bottom panel).

p300 condensates sequester nucleosomal substrates. Besides auto-acetylation, p300 catalyzes acetylation of lysine residues of 


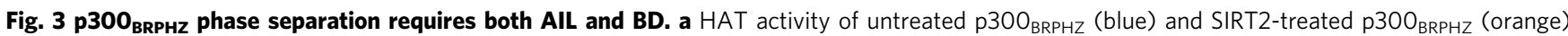
measured by a fluorometric assay. Reactions were started by the addition of acetyl-CoA and quenched by flash-freeze at indicated time points. Data are presented as mean values $\pm S D$; error bars represent SD from triplicate measurements. b Phase separation of SIRT2-treated $\mathrm{p} 30 \mathrm{O}_{\mathrm{BRPHZ}}$. The reaction mixture contained $10 \mu \mathrm{M} \mathrm{p} 300_{\mathrm{BRPHZ}}$ and $12 \%$ PEG. Addition of Acetyl-CoA led to the disassembly of the p300 $\mathrm{BRPHZ}_{\mathrm{B}}$ droplets. c Representative images of p300 $0_{\mathrm{BRPHZ}}$ samples from $(k)$ after incubation for 2 min under a microscope. $\mathbf{d}$ Quantification of droplets counted in images acquired for the sample in (c). The number of droplets was counted in five non-overlapping $50 \mu \mathrm{m} \times 50 \mu \mathrm{m}$ square regions and the mean value was plotted. Error bar represents SD. e Schematics of the $\mathrm{p} 300_{\mathrm{BRPHZ}}$ phase separation mechanisms that can occur simultaneously. Multivalent 'in trans' interactions between HAT and deacetylated AIL and/or BD and acetylated lysines outside AIL can promote the formation of condensates. $\mathbf{f}, \mathbf{g}$ Representative images of WT p300 $\mathrm{BRPHZ}_{\mathrm{B}}(\mathbf{f})$ and N1132A mutant (g) samples (from at least three replicates) containing $13 \mu \mathrm{M}$ protein and $12 \%$ PEG in a $50 \mu \mathrm{m} \times 50 \mu \mathrm{m}$ square region on cover slides

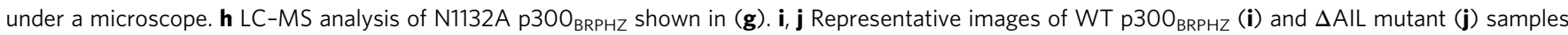
(from at least three replicates) containing $13 \mu \mathrm{M}$ protein and $12 \%$ PEG in a $50 \mu \mathrm{m} \times 50 \mu \mathrm{m}$ square region on cover slides under a microscope. $\mathbf{k} \mathrm{LC}-\mathrm{MS}$ analysis of $\triangle$ AIL $\mathrm{p} 30 \mathrm{O}_{\mathrm{BRPHZ}}$ shown in $(\mathbf{j})$. Source data are provided in the Source Data file.

a

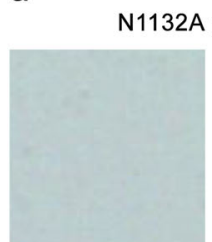

- SIRT2 $b$

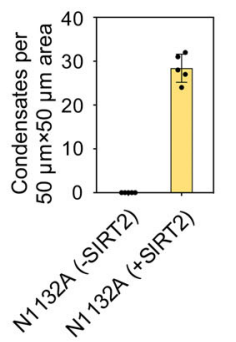

$c$

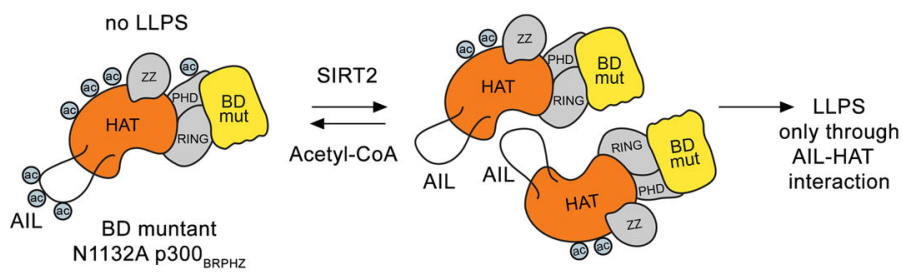

$d$

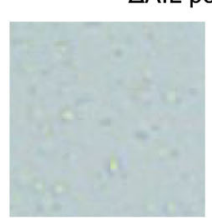

- SIRT2

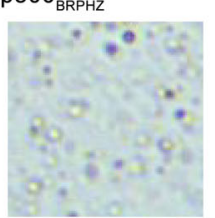

+ SIRT2 e

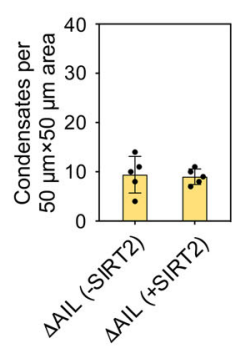

$f$

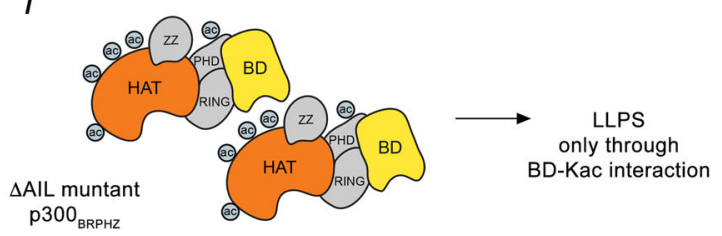

$g$

D1399A p300 ${ }_{\text {BRPHZ }}$

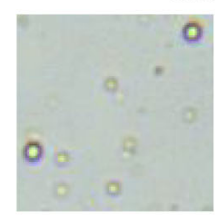

- SIRT2

k

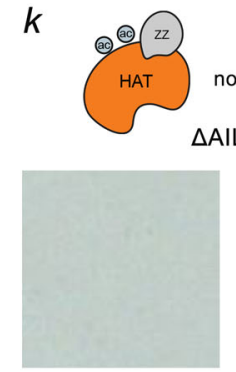

- SIRT2

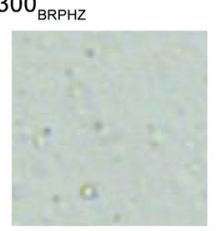

+ SIRT2

h
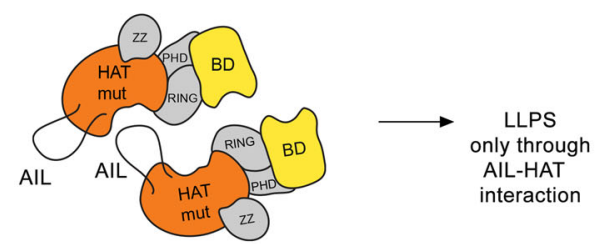

no LLPS

$\triangle$ AIL p $300_{\mathrm{Hz}}$

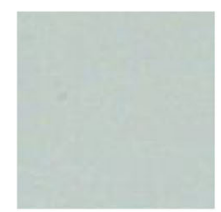

+ SIRT2
I

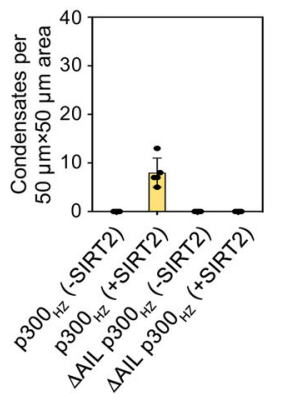

i

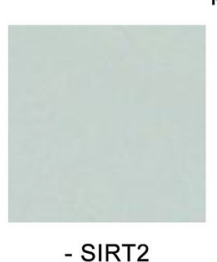

$\mathrm{p} 300_{\mathrm{Hz}}$

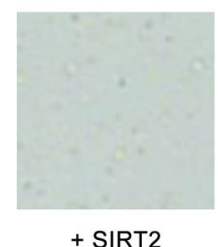

j

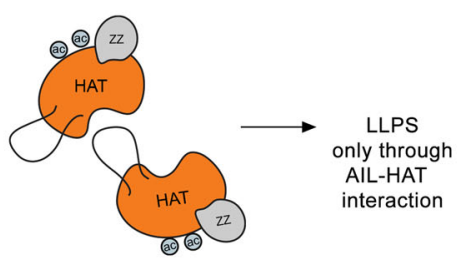

$m$

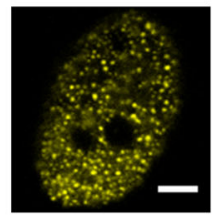

N1132A YFP-p300 FL

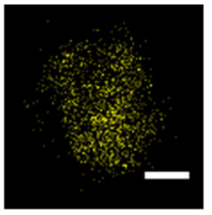

$\Delta$ AIL YFP-p300 
Fig. 4 The molecular mechanisms of $\mathbf{p} 300_{\text {BRPHZ }}$ phase separation. a Representative images of untreated or SIRT2-deacetylated N1132A p300 ${ }_{B R P H Z}$ samples containing $13 \mu \mathrm{M}$ protein and $12 \%$ PEG in a $50 \mu \mathrm{m} \times 50 \mu \mathrm{m}$ square region on cover slides under the microscope. $\mathbf{b}$ Quantification of droplets counted in images acquired for the sample in (a). The number of droplets was counted in five non-overlapping $50 \mu \mathrm{m} \times 50 \mu \mathrm{m}$ square regions and the mean value was plotted. Error bar represents SD. c Schematic of the N1132A P300 ${ }_{\text {BRPHZ }}$ phase separation via the intermolecular interaction between the HAT domain and deacetylated AIL. d Representative images of untreated or SIRT2-deacetylated $\Delta$ AIL p $300_{\text {BRPHZ }}$ samples containing $13 \mu \mathrm{M}$ protein and $12 \%$ PEG in a $50 \mu \mathrm{m} \times 50 \mu \mathrm{m}$ square region on cover slides under a microscope. e Quantification of droplets counted in images acquired for the sample in (d). The number of droplets was counted in five non-overlapping $50 \mu \mathrm{m} \times 50 \mu \mathrm{m}$ square regions and the mean value was plotted. Error bar represents SD. $\mathbf{f}$

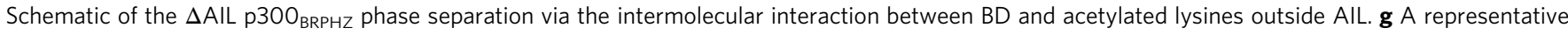
image of purified D1399A p $300_{\mathrm{BRPHZ}}$ sample (from at least three replicates) containing $13 \mu \mathrm{M}$ protein and $12 \%$ PEG in a $50 \mu \mathrm{m} \times 50 \mu \mathrm{m}$ square region on cover slides under the microscope. $\mathbf{h}$ Schematic of the catalytically inactive D1399A p300 ${ }_{\mathrm{BRPHz}}$ phase separation via the intermolecular interaction between the HAT domain and unmodified AIL. i Representative images of untreated or SIRT2-deacetylated WT p $300_{\mathrm{HZ}}$ samples containing $13 \mu \mathrm{M}$ protein and $12 \%$ PEG in a $50 \mu \mathrm{m} \times 50 \mu \mathrm{m}$ square region on cover slides under the microscope. $\mathbf{j}$ Schematic of the $\mathrm{p} 30 \mathrm{O}_{\mathrm{Hz}}$ phase separation via the intermolecular interaction between the HAT domain and deacetylated AIL. $\mathbf{k}$ Representative images of untreated or SIRT2-deacetylated $\Delta$ AIL p300 $\mathrm{Hz}$ samples containing $13 \mu \mathrm{M}$ protein and $12 \%$ PEG in a $50 \mu \mathrm{m} \times 50 \mu \mathrm{m}$ square region on cover slides under the microscope. I Quantification of droplets counted in images acquired for the samples in $(\mathbf{i}, \mathbf{k})$. The number of droplets was counted in five non-overlapping $50 \mu \mathrm{m} \times 50 \mu \mathrm{m}$ square regions and the mean value was plotted. Data are presented as mean values \pm SD; the error bar represents SD. $\mathbf{m}$ Representative images of HeLa cells (from at least three replicates) expressing N1132A and $\Delta$ AIL mutants of YFP-p300 FL. Scale bar, $5 \mu \mathrm{m}$.

histone proteins in nucleosomes, particularly at $\mathrm{H} 3 \mathrm{~K} 18$ and H3K27 sites $22,23,25$. We, therefore, tested whether p300 droplets could concentrate nucleosomal components, such as DNA and histone tails. We monitored the recruitment of fluorescein (FAM)-labeled histone $\mathrm{H} 3$ tail (FAM-H3, residues 1-12 of H3) and FAM-labeled $37 \mathrm{bp}$ double-stranded DNA (FAM-DNA) to p300 ${ }_{\text {BRPHZ }}$ droplets using confocal microscopy (Fig. 6a and Supplementary Fig. 3b). The p300 condensates exhibited bright fluorescence when incubated with FAM-DNA or FAM-H3, but not when incubated with the control FAM. The recruitment of histone $\mathrm{H} 3$ tail to condensates was due to binding of the $\mathrm{ZZ}$ domain of p300 to the unmodified H3 tail ${ }^{25}$, however, p300 and CBP were thought to not contact DNA themselves ${ }^{34}$. We noticed lower background fluorescence when p300 condensates were incubated with FAM-DNA compared to FAM-H3, which suggests that $\mathrm{p} 300_{\mathrm{BRPHZ}}$ concentrates better and therefore binds stronger to DNA than to the histone $\mathrm{H} 3_{1-12}$ peptide. Overall, these results demonstrate that $\mathrm{p} 300_{\mathrm{BRPHZ}}$ condensates can sequester nucleosomal components like histone $\mathrm{H} 3$ tail and DNA from the surrounding environment.

Can p300 bind DNA and is this reaction mediated by p300 self-acetylation? We tested the association of untreated and SIRT2-treated p300 $\mathrm{BRPHZ}$ with 601 DNA (147 bp) by electrophoretic mobility shift assay (EMSA). The SIRT2-treated p300 ${ }_{\text {BRPHZ }}$, containing on average 4 Kac outside the AIL, strongly bound to 601 DNA with an apparent $K_{\mathrm{d}}$ of $\sim 0.3 \mu \mathrm{M}$ (Fig. $6 \mathrm{~b}$ ). In contrast, untreated $\mathrm{p} 300_{\mathrm{BRPHZ}}$, which contains on average $\sim 16$ Kac including in the AIL, showed much weaker binding to 601 DNA in the same condition (Fig. 6c). DNA binding of p300 ${ }_{\mathrm{BRPHZ}}$, therefore, is directly regulated by its acetylation level, as acetylation neutralizes the positive charge of the unmodified lysine side chain, leading to a decrease in binding to the negatively charged DNA. We also found that auto-acetylation regulates $\mathrm{p} 300_{\mathrm{BRPHZ}}$ association with nucleosomes. While SIRT2treated $\mathrm{p} 300_{\mathrm{BRPHZ}}$ bound to the reconstituted nucleosome core particle (NCP) with a low micromolar $K_{\mathrm{d}}$ in EMSA assays (Fig. 6d), the association of untreated $\mathrm{p} 300_{\mathrm{BRPHZ}}$ with NCP was notably compromised (Fig. 6e). Collectively, these data suggest that the phase transition and nucleosome binding functions of p $300_{\mathrm{BRPHZ}}$ are linked to and regulated by its auto-acetylation.

The results described above may suggest that DNA and the HAT domain compete for the same hypoacetylated AIL, which could lead to a decrease in phase separation ability of p300 ${ }_{\mathrm{BRPHZ}}$. However, the addition of an equimolar amount of NCP to p300 $0_{\text {BRPHZ }}$ stimulated the formation of more and larger droplets, whereas NCPs alone did not phase separate under the same condition (Fig. 6f). These data imply that even when the AIL binds to DNA and is, therefore, less available to contribute to the droplet formation through the HAT-AIL mechanism, the intramolecular association through the BD-Kac mechanism is sufficient to maintain phase separation.

Catalytic HAT activity of p300 is decreased in condensates. We examined the effect of phase separation on enzymatic activity and histone substrate selectivity of p300 $\mathrm{BRPHZ}$. Because phase separation conditions can promote enzymatic reactions owing to higher local concentrations of both the enzyme and substrate ${ }^{44}$, we initially thought that p300 HAT activity would also be elevated due to increased local concentration of $\mathrm{p} 300_{\mathrm{BRPHZ}}$ and the substrate, either $\mathrm{p} 300_{\mathrm{BRPHZ}}$ and/or NCP inside the droplets. On the other hand, the intermolecular AIL-HAT interaction, which leads to the formation of p300 condensates, physically blocks the active site of the HAT domain and thus would lead to a reduction in the enzymatic activity, similar to what was observed for the TOR kinase ${ }^{45}$. In addition to sterically occluding the substrate-enzyme complex formation, the crowed viscous environment in condensates slows diffusion of substrates and products, which results in the reduced catalytic activity of enzymes ${ }^{46,47}$.

To compare the HAT activity in solution and phase-separated droplets, we first monitored the self-acetylation of p $300_{\mathrm{BRPHZ}}$. As shown in Fig. 6g, the addition of PEG to the SIRT2-treated p300 $0_{\mathrm{BRPHZ}}$, which does not phase separate because of low protein concentration $(0.2 \mu \mathrm{M})$, had essentially no effect on the change in the fluorescence signal (due to CoA release) over time, suggesting that the presence of PEG did not alter the catalytic activity of p300 $0_{\text {BRPHZ }}$ (Fig. 6g). In contrast, the change in fluorescence was slower in the PEG-induced phase-separated suspension (Fig. 6h, red line) or in the supernatant after the droplets were spun down (Fig. 6i) compared to the change in fluorescence in the solution without droplets (Fig. 6h, blue line). These data indicate that autoacetylation is inhibited when $\mathrm{p} 300_{\mathrm{BRPHZ}}$ forms condensates.

We next measured the acetyltransferase activity of p $300_{\mathrm{BRPHZ}}$ on reconstituted nucleosomes in homogenous solutions and under the phase separation condition. SIRT2-treated (containing $\sim 12 \mathrm{Kac}$ ) p300 $0_{\mathrm{BRPHZ}}$ and untreated (containing $26 \mathrm{Kac}$ ) p300 $0_{\mathrm{BRPHZ}}$ were mixed with $\mathrm{NCP}$ at a 1:1 ratio, and after the addition of acetyl-CoA, the CoA release was monitored at indicated time points. The reactions with NCP as a substrate (Fig. 6j, blue and orange bars, and Supplementary Fig. 4a) were completed within $2 \mathrm{~min}$ for both SIRT2-treated and untreated p $300_{\text {BRPHZ }}$ and were much faster than $\mathrm{p} 300_{\mathrm{BRPHZ}}$ autoacetylation 

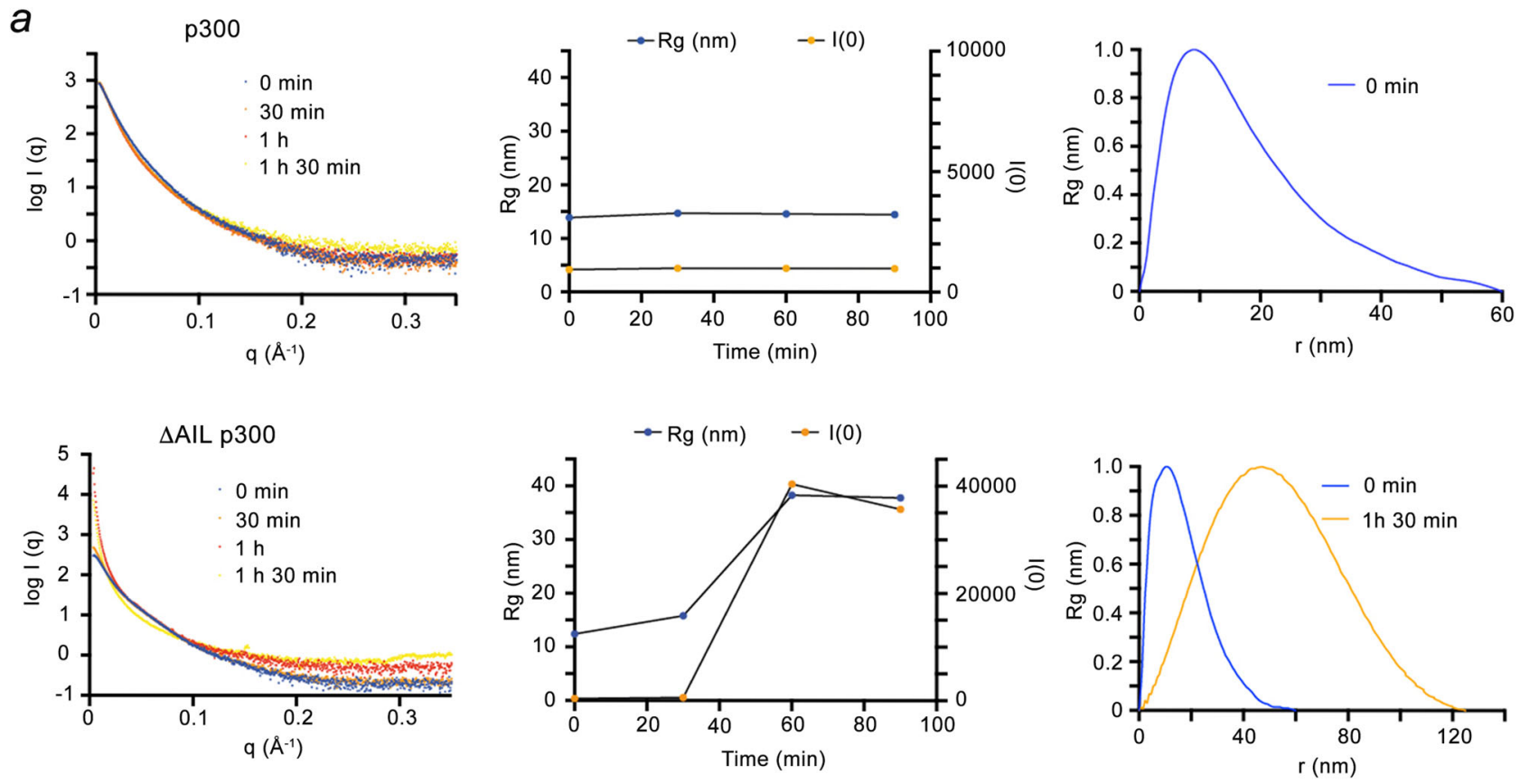

$b$

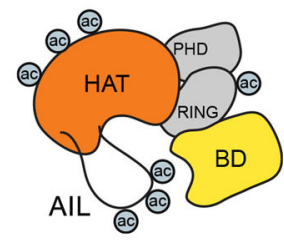

WT p $300_{\text {BRPH }}$

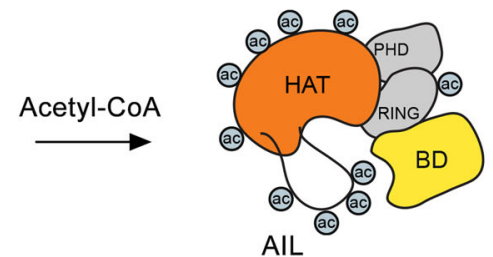

AIL

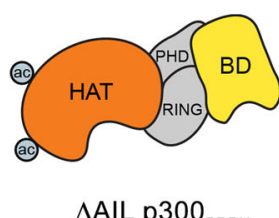

Acetyl-CoA

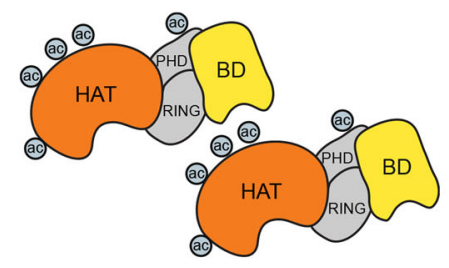

Fig. $5 \Delta$ AIL $\mathbf{p 3 0 0}_{\mathbf{3 4 0 - 2 0 9 4}}$ interacts 'in trans' in solution. a Small angle X-ray scattering (SAXS) data collected for WT (upper panel) and $\Delta$ AIL (lower panel) $\mathrm{p} \mathrm{O}_{340-2094}$ upon addition of acetyl-CoA at indicated time points including 0 min (blue) and 90 min (orange). SAXS intensity (left), Rg plots (middle), and $P(r)$ functions (right) determined from the experimental scattering data were shown. $\mathbf{b}$ Schematics of the WT and $\triangle A I L ~ p 30 O_{B R P H}$ intramolecular and intermolecular interactions between BD and acetylated lysines.

(Fig. 3a). The HAT reaction was also carried out in a suspension of PEG-induced droplets of SIRT2-treated $\mathrm{p} 300_{\mathrm{BRPHZ}}$ and NCP. Again, as in the case of autoacetylation (Supplementary Fig. 4b, gray bars), the apparent HAT activity of $\mathrm{p} 300_{\mathrm{BRPHZ}}$ in the phaseseparated condensates was decreased compared to the HAT

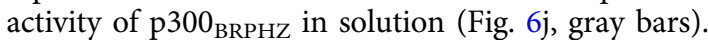

To assess the histone substrate selectivity of $\mathrm{p} 300_{\mathrm{BRPHZ}}$, we monitored acetylation of NCP by western blot using antibodies against H3K27ac, H3K9ac, and H3K4ac (Fig. 7a-d and Supplementary Fig. 5). We found that either SIRT2-treated or

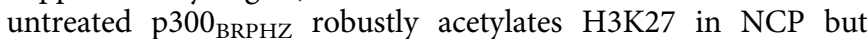
produces $\mathrm{H} 3 \mathrm{~K} 9 \mathrm{ac}$ and $\mathrm{H} 3 \mathrm{~K} 4 \mathrm{ac}$ to a lesser degree, which is in agreement with our previous findings ${ }^{25}$ and with the data shown in Fig. $6 j-$ the reaction was fast and completed in $2 \mathrm{~min}$. The selectivity of $\mathrm{p} 300_{\mathrm{BRPHZ}}$ toward the $\mathrm{H} 3 \mathrm{~K} 27$ site was conserved in the suspension of SIRT2-treated p $300_{\mathrm{BRPHZ}}$ droplets, however, again, the rate of NCP acetylation was decreased (Fig. 7b, d, yellow line). These data further substantiate a reduction in the catalytic activity of p300 $\mathrm{BRPHZ}_{\mathrm{H}}$ upon formation of the condensates.

p300 condensates preferably localize to chromatin regions marked by H3K27me3. p300 often binds to enhancers and promoters enriched in active mono- and trimethylated $\mathrm{H} 3 \mathrm{~K} 4$ (H3K4me1 and H3K4me3, respectively) marks, acetylating histones, and stimulating gene transcription. However, a pool of p300/CBP with a suppressed HAT activity has been shown to associate with poised and silent genomic regions marked by the repressive modification $\mathrm{H} 3 \mathrm{~K} 27 \mathrm{me} 3^{25,29-31,48,49}$. Indeed, ChIPseq analysis of the H1299 cells expressing Flag-tagged WT

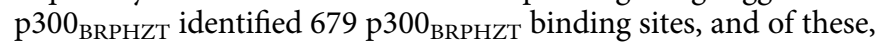



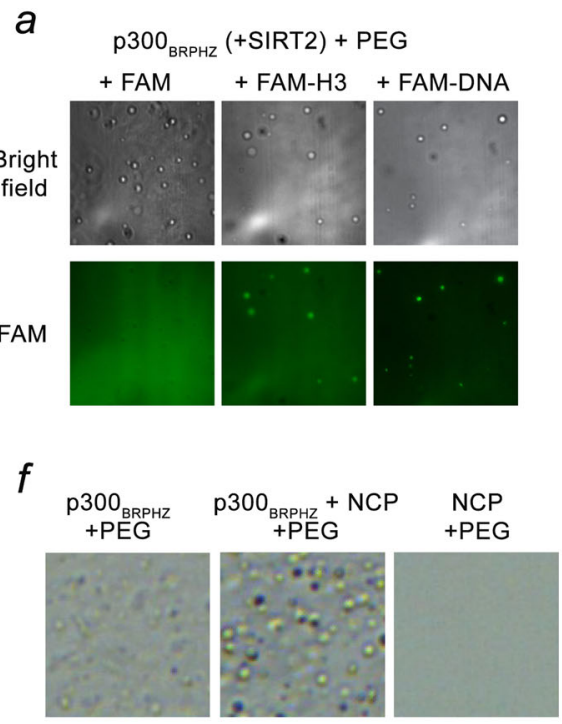

$b$

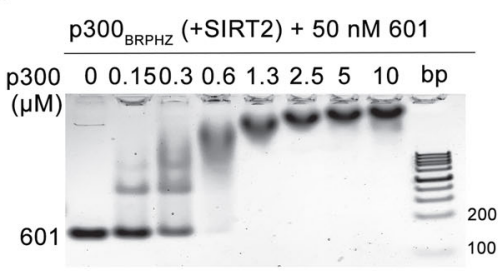

d

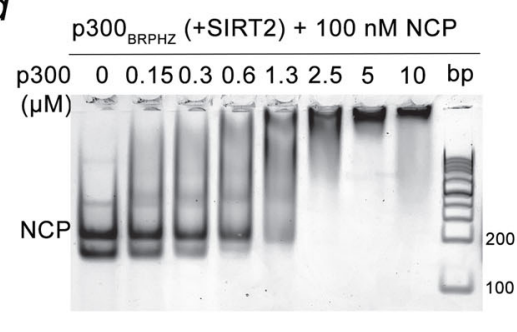

C

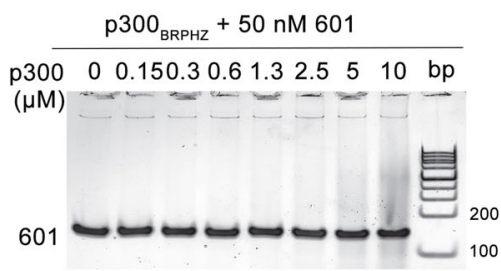

e

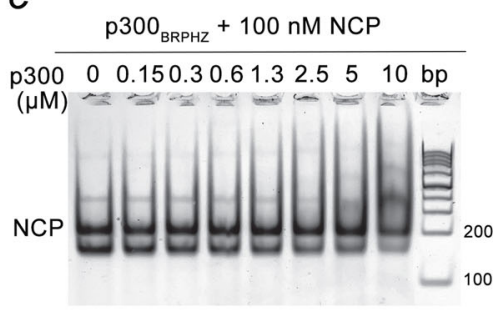

j $\quad \mathrm{p}^{2} 0 \mathrm{O}_{\mathrm{BRPH}}+\mathrm{NCP}$

- -SIRT2 =+SIRT2 =+SIRT2 +PEG

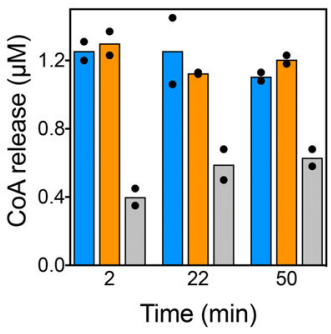

$g$

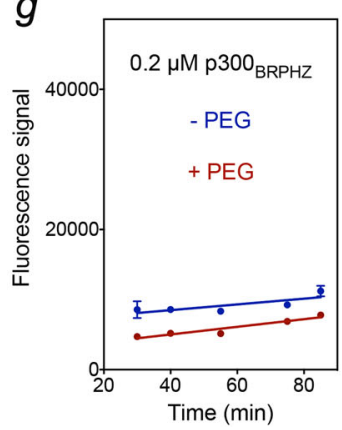

$h$

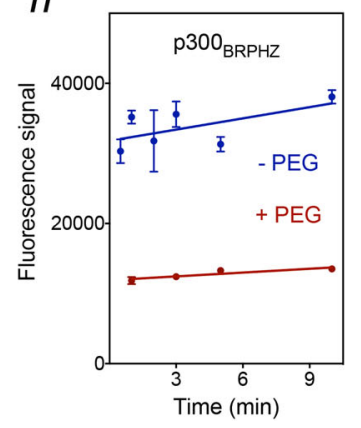

i

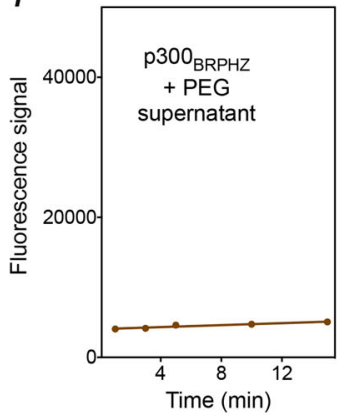

Fig. 6 Acetylation regulates the association of $\mathbf{p} \mathbf{3 0 0}$ with DNA and the nucleosome. a Representative confocal images of phase-separated SIRT2-treated p $300_{\text {BRPHZ }}$ condensates with FAM control (left), FAM-labeled H3 $3_{1-12}$ peptide (middle), and FAM-labeled DNA (right). A $50 \mu \mathrm{m} \times 50 \mu \mathrm{m}$ square region is

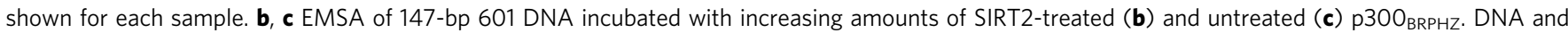
protein concentrations are shown above the gel image. d, e EMSA of the reconstituted nucleosome incubated with increasing amounts of SIRT2-treated (d) and untreated (e) $\mathrm{p} 300_{\mathrm{BRPHZ}}$. Nucleosome core particle (NCP) and protein concentrations are shown above the gel image. f Phase separation of SIRT2treated $\mathrm{p} 300_{\mathrm{BRPHZ}}$ alone (left), p300 $\mathrm{BRPHz}$ incubated with NCP (middle) and NCP alone (right) in a buffer containing $12 \%$ PEG. A $50 \mu \mathrm{m} \times 50 \mu \mathrm{m}$ square region is shown for each sample. Experiments in a-f were performed in at least two replicates. $\mathbf{g}$ HAT activity of diluted $(0.2 \mu \mathrm{M})(\mathbf{g})$ and concentrated $(10$ $\mu \mathrm{M}$ ) (h) SIRT2-deacetylated $\mathrm{p} 30 \mathrm{O}_{\mathrm{BRPHZ}} \pm$ PEG was measured by a fluorometric assay. i HAT activity of SIRT2-deacetylated $\mathrm{p} 30 \mathrm{O}_{\mathrm{BRPHZ}}$ in supernatant measured by a fluorometric assay. Droplets in the phase-separated $\mathrm{p} 300_{\mathrm{BRPHZ}}$ sample were spun down for 10 min, and the supernatant was transferred to another tube. For all experiments in ( $\mathbf{g}-\mathbf{i})$, reactions were quenched by adding $6 \mathrm{M}$ guanidine hydrochloride. The relative reaction rates (slope values) are $0.020(-P E G)$ and $0.027(+P E G)$ in ( $\mathbf{g}), 0.27(-P E G)$ and $0.09(+P E G)$ in (h), and 0.035 in (i). Data in $\mathbf{g}, \mathbf{h}$ are presented as mean values \pm SD; error bars represent SD from triplicate measurements. $\mathbf{j}$ HAT activity of untreated $\mathrm{p} 300_{\mathrm{BRPHZ}}$ (blue), SIRT2-treated p300 BRPHZ (orange), and SIRT2-treated phaseseparated $\mathrm{p} 30 \mathrm{O}_{\mathrm{BRPHZ}}$ droplet suspension (gray) on NCP measured by a fluorometric assay. Data are presented as mean values from duplicate measurements.

117 p $300_{\text {BRPHZT }}$ binding sites were also enriched in H3K27ac and $\mathrm{H} 3 \mathrm{~K} 18 \mathrm{ac}$ - the primary products of acetylation by p300 (Fig. 7e). 76 p300 ${ }_{\text {BRPHZT }}$ binding sites however were enriched in $\mathrm{H} 3 \mathrm{~K} 27 \mathrm{me} 3$. The notable absence of acetylation of $\mathrm{H} 3 \mathrm{~K} 18$ at these sites suggests that the HAT activity of $\mathrm{p} 300_{\mathrm{BRPHZT}}$ is decreased when p300 colocalizes with H3K27me3.

Because the HAT activity of p300 is decreased in the phaseseparated condensates, we examined whether the condensates could select for chromatin modifications in HeLa cells using immunofluorescence. As shown in Fig. 8, the YFP-p300 $\mathrm{FL}$ condensates co-localize to a higher degree with the regions enriched in H3K27me3 with a Pearson's correlation coefficient (Pearson's $R$ ) of $0.45 \pm 0.09$, but co-localization with H3K4me3 was less pronounced (Pearson's $R$ of $0.37 \pm 0.09$ ), and even lower level of co-localization was observed with $\mathrm{H} 3 \mathrm{~K} 9 \mathrm{me} 3$ (Pearson's $R$ of $0.25 \pm 0.05$ ). Together, these data suggest that a pool of p300 condensates has a preference to localize to the chromatin regions containing transcriptionally repressive $\mathrm{H} 3 \mathrm{~K} 27 \mathrm{me} 3$ modification as compared to the regions containing transcriptionally active modification $\mathrm{H} 3 \mathrm{~K} 4 \mathrm{me} 3$ or the heterochromatin mark H3K9me3.

It is becoming increasingly clear that the LLPS phenomenon and the assembly of membraneless condensates by biological macromolecules in the nucleus play a crucial role in numerous cellular processes. Formation of condensates allows for efficient separation of the nuclear compartments in a spatiotemporal manner and/or concentration of the macromolecules to regulate, activate or reduce their functions. Although the phase separation mechanisms in the nucleus are currently a subject of intense studies, multivalent weak contacts involving IDRs of macromolecules have been widely acknowledged as a driving force for the formation of biomolecular condensates. In this work, we show 

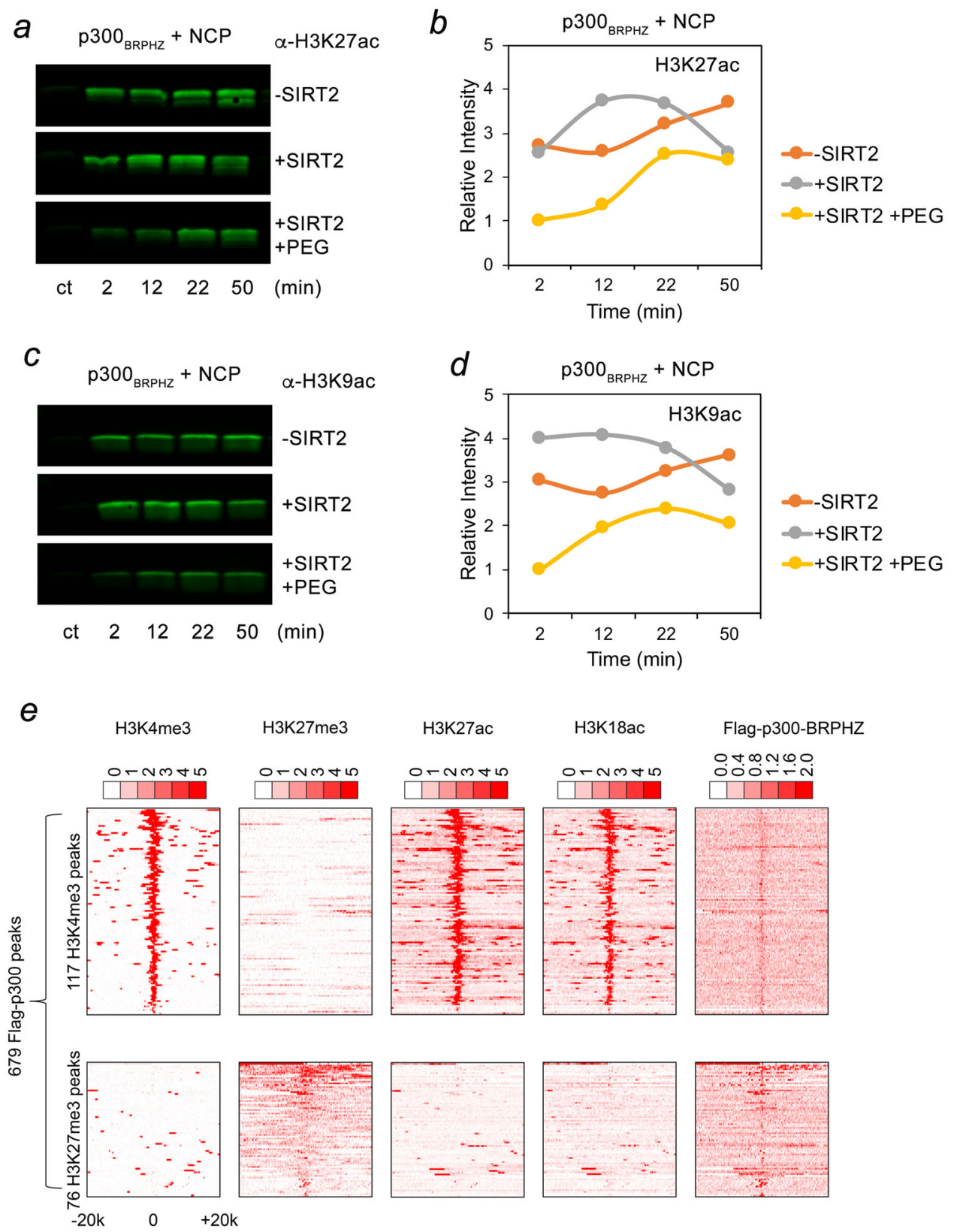

Fig. 7 A pool of p300 co-localizes with H3K27me3. a-d H3K27ac and H3K9ac western blot analysis of the reaction mixtures containing p300 ${ }_{B R P H Z}$ and an equimolar amount of NCP as a substrate. Reactions were quenched by rapid freezing and the addition of SDS-loading buffer at indicated time points. The intensity of bands is quantified, normalized to the +SIRT2/+PEG band at 2 min, and shown in (b) and (d). e Heatmaps of H3K4me3, H3K27me3, H3K27ac,

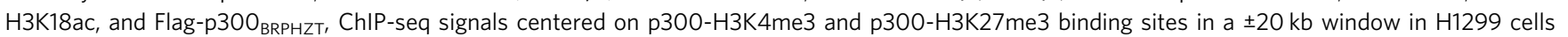

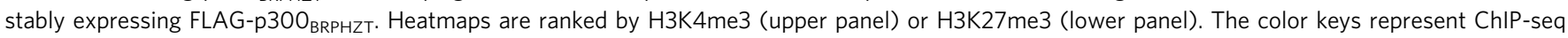
densities normalized to total reads.

that the major human acetyltransferase p300 forms liquid condensates in the nucleus and this function depends rather on the structured catalytic core of p300, including the HAT domain and its AIL, and BD. We demonstrate that hyperacetylation of the p300 catalytic core, particularly of AIL, decreases the phase separation ability, and that p300 utilizes two distinct molecular mechanisms to assemble the condensates, which rely on intermolecular 'in trans' HAT-AIL and BD-(Kac outside AIL) interactions. Furthermore, we found that the catalytic HAT activity of p300 is decreased in the phase-separated droplets, which is likely due to steric blocking of the HAT active site.

Our data suggest a model for compartmentalization and concentration of p300 with reduced catalytic activity. p300/CBP is often associated with transcriptional activation and occupies gene promoters and enhancer elements but has also been reported to localize to the repressive sites, particularly those enriched in $\mathrm{H} 3 \mathrm{~K} 27 \mathrm{me} 3^{29,30}$. These sites are characterized by overall low acetylation of histones, and since $\mathrm{H} 3 \mathrm{~K} 27 \mathrm{me} 3$ does not preclude binding of $\mathrm{p} 300 / \mathrm{CBP}$ to the $\mathrm{H} 3$ tail, it was proposed that the HAT activity is blocked at such sites ${ }^{31,49}$. The mechanism of this blockage remains unclear. A few concepts have been put forth to explain the decrease in the p300/CBP catalytic activity, especially on histone lysine residues other than $\mathrm{H} 3 \mathrm{~K} 27$, as methylation of H3K27 obviously prevents its acetylation. These include regulation through phosphorylation or SUMOylation of p300/CBP 34,50 and rapid degradation of $\mathrm{p} 300$. Our finding that a large portion of 
a
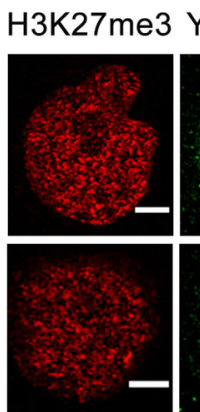

C

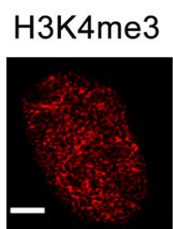

YFP-p300

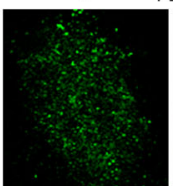

merge

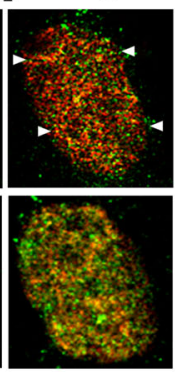

e

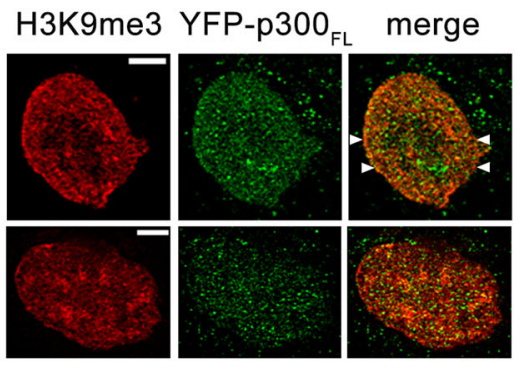

$b$

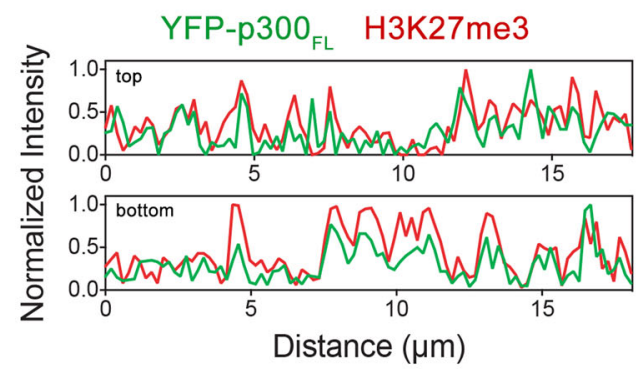

$d$

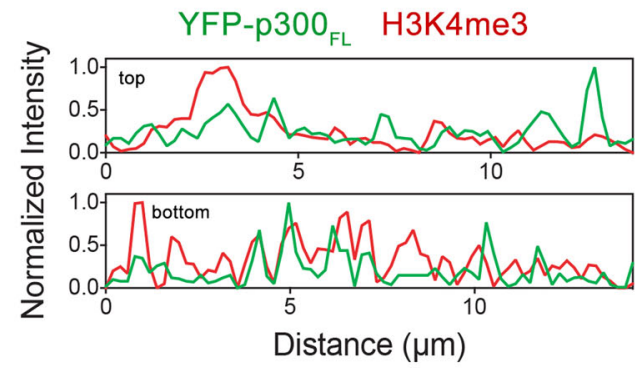

f

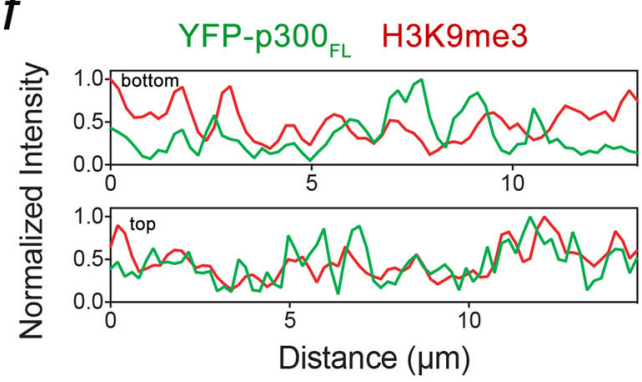

$g$

\begin{tabular}{|c|c|}
\hline Histone PTM & Pearson's R value \\
\hline H3K27me3 & $0.45+/-0.09$ \\
\hline H3K4me3 & $0.37+/-0.09$ \\
\hline H3K9me3 & $0.25+/-0.05$ \\
\hline
\end{tabular}

Fig. 8 p300 condensates show preference for co-localization with H3K27me3. a-f Nuclear localization of the YFP-p300 ${ }_{\mathrm{FL}}$ condensates with indicated posttranslational histone modifications (PTMs) in HeLa cells were examined by immunofluorescence. The cells were fixed and probed with antiH3K27me3 (a), anti-H3K4me3 (c), and anti-H3K9me3 (e) antibodies. Representative images are shown in the left panels: green, YFP-p300 FL; red, PTMs; yellow, overlap of fluorescence signals of YFP-p300 FL and PTMs. The ImageJ software was used to plot fluorescence signal intensities of YFP-p300 $\mathrm{FL}_{\mathrm{FL}}$ and PTMs in the nuclei along the line (from left to right) indicated by white arrowheads (b, d, f). Scale bar, $5 \mu \mathrm{m}$. $\mathbf{g} \mathrm{A}$ table of Pearson's $R$ values calculated using the entire cell nuclei ( $n=3$ cells examined for each mark). Data are presented as mean values \pm SD. Pearson's $R$ values range between +1 (perfect positive correlation) and -1 (inverse correlation).

p300 condensates co-localizes with chromatin regions enriched in $\mathrm{H} 3 \mathrm{~K} 27 \mathrm{me} 3$ suggests an alternative mechanism. The p300 condensates can act as a storage pool of the protein with reduced HAT activity, allowing p300 and possibly other elements of the transcriptional machinery to be compartmentalized and concentrated at the repressed chromatin sites. The formation of p300 condensates through blocking the catalytic site of the HAT domain provides a mechanism by which the enzymatic activity of p300 can be downregulated. Similar downregulation of the enzymatic activity through phase transition has been reported for the target of rapamycin (TOR) serine/threonine protein kinase in the TORC1 complex ${ }^{45}$. The formation of the TORC1 foci results in steric occlusion of the TOR active site, subsequently leading to the inhibition of the catalytic activity ${ }^{45}$. To better understand the p300-dependent activation of gene transcription, it will be essential in future studies to delineate and visualize by singlemolecule imaging ${ }^{51}$ the precise contacts between p300 and the repressed chromatin regions. 


\section{Methods}

Cell culture, transfection, and imaging. WT full-length YFP-p300 plasmids were transfected into HeLa cells in a 3.5-cm-diameter tissue culture dish by Lipofectamine 3000 (Life Technology, L3000-075) using manufacturer instructions. The cells were cultured in DMEM (company) supplemented with 10\% fetal bovine serum (company, need double-check) for $36 \mathrm{~h}$. The cell culture medium was replaced with the live-cell imaging medium and maintained at $37^{\circ} \mathrm{C}$ using a heater controller. The p300 BD-Ring-PHD-HAT-ZZ_TAZ2 region (BRPHZT, aa 1024-1830) was cloned into a pTripZ-YFP vector. To establish a stable cell line, viral titer harvested from HEK293T cells was used to infect HeLa cells with fusion gene. HEK293T cells were seeded in a 10-cm dish to reach $90-100 \%$ confluency the following day. The cells were then transfected using calcium phosphate precipitation $\left(21.0 \mu \mathrm{g}\right.$ psPAX2, 10.5, $21.0 \mu \mathrm{g}$ of pTripZ-YFP-p300, $250 \mathrm{mM} \mathrm{CaCl}_{2}$, and $1 \times$ Hank's balanced salt solution) and incubated for $12 \mathrm{~h}$ at $37^{\circ} \mathrm{C}$ and $5 \% \mathrm{CO}_{2}$. Cells were washed twice with culture medium and then incubated in culture medium for $48 \mathrm{~h}$. Viral titer was harvested from HEK293T cell culture medium and was spun at $1000 \times g$ for $5 \mathrm{~min}$ to remove any cell debris. This was added to a single-cell suspension of HeLa cells supplemented with $8 \mu \mathrm{g} / \mathrm{mL}$ Polybrene (SIGMA, H9268) and was mixed. Solution of virus and HeLa cells was plated evenly into a $10-\mathrm{cm}$ dish and incubated for $12 \mathrm{~h}$. Culture medium of infected cells was then replaced, and cells were maintained.

FRAP experiments. FRAP imaging was performed using a Zeiss LSM 700 Observer as described previously ${ }^{16}$. Briefly, two images were taken before photobleaching, and 15-30 images were taken with $10 \mathrm{~s}$ intervals immediately after photobleaching. The images were analyzed using ImageJ. Fluorescence intensities were normalized to the signal before photobleaching to obtain the fluorescence recovery.

Protein expression and purification. The construct of human full-length p300 protein-containing BD-RING-PHD-HAT-ZZ region (BRPHZ, amino acids 1035-1720) was cloned in the pGEX-6P-1 vector and expressed in BL21 (RIL) cells as previously described ${ }^{25}$. Protein production was induced with $0.1 \mathrm{mM}$ IPTG and cultured overnight at $16^{\circ} \mathrm{C}$ in Luria broth (LB) medium. The GST-tagged proteins were purified on Pierce glutathione agarose beads (Thermo-Fisher) in $20 \mathrm{mM}$ Tris-HCl (pH 7.5), $500 \mathrm{mM} \mathrm{NaCl}$, and $3 \mathrm{mM}$ DTT. The GST tag was cleaved overnight at $4{ }^{\circ} \mathrm{C}$ with PreScission proteases. The SIRT2 (38-356) construct fused with His6x-SUMO was obtained from Addgene (addgene ID:102622). SIRT2 protein was expressed and purified using a standard protocol. In brief, the cells were disrupted by sonication, and the cell lysate was spun down to remove the debris. The supernatant was loaded to a nickel column (Histrap, GE Healthcare) pre-equilibrated with a buffer containing $50 \mathrm{mM}$ Tris- $\mathrm{HCl} \mathrm{pH} 7.5$ and $500 \mathrm{mM}$ $\mathrm{NaCl}$. The SIRT2 protein was eluted with a $0-500 \mathrm{mM}$ linear gradient of imidazole. The deacetylation reaction was carried out by incubating purified p300 WT and mutant proteins with SIRT2 overnight in the cold room in a buffer containing 20-50 mM Tris ( $\mathrm{pH} 7.5$ ), $300 \mathrm{mM} \mathrm{NaCl}, 5 \mathrm{mM} \mathrm{MgCl}, 2 \mathrm{mM} \mathrm{NAD}$, and $2 \mathrm{mM}$ DTT. Deacetylated p300 proteins were further purified by size-exclusion chromatography and concentrated in Millipore concentrators.

Trypsin digestion of acetylated p300 proteins. Purified p300 3 BRPHZ $(40-50 \mu \mathrm{g})$ with or without SIRT2 treatment was denatured, reduced, and alkylated in $5 \%(\mathrm{w} /$ v) sodium dodecyl sulfate (SDS), $10 \mathrm{mM}$ tris (2-carboxyethyl) phosphine hydrochloride (TCEP-HCl), $40 \mathrm{mM}$ 2-chloroacetamide, $50 \mathrm{mM}$ Tris $\mathrm{pH} 8.5$ and boiled at $95^{\circ} \mathrm{C}$ for $10 \mathrm{~min}$. Samples were prepared for mass spectrometry analyses using the SP3 method ${ }^{52}$. Carboxylate-functionalized speedbeads (GE Life Sciences) were added to protein samples. Acetonitrile was added to $80 \%(\mathrm{v} / \mathrm{v})$ to precipitate protein and bind it to the beads. The protein-bound beads were washed twice with $80 \%$ (v/v) ethanol and twice with $100 \%$ acetonitrile. Lys-C/Trypsin mix (Promega) was added for 1:50 protease to protein ratio in $50 \mathrm{mM}$ Tris $\mathrm{pH} 8.5$ and incubated rotating at $37^{\circ} \mathrm{C}$ overnight. To clean up tryptic peptides, acetonitrile was added to $95 \%(\mathrm{v} / \mathrm{v})$ to precipitate and bind peptides to the beads. One wash with $100 \%$ acetonitrile was performed and tryptic peptides were eluted twice with $1 \%(\mathrm{v} / \mathrm{v})$ trifluoroacetic acid (TFA), 3\% (v/v) acetonitrile in water. Eluate was dried using a speed-vac rotatory evaporator.

Liquid chromatography and tandem mass spectrometry (LC-MS/MS) analysis. For estimations of intact protein masses, untreated and SIRT2-treated WT and mutant p300 protein samples were resolved using a Waters AQCUITY UPLC. Proteins were diluted to final concentration of $0.3 \mu \mathrm{g} / \mu \mathrm{L}$ using Buffer A $(0.1 \%$ formic acid in water), of which $3 \mu \mathrm{g}$ of protein was loaded onto a Waters ACQUITY UPLC Protein BEH C4 Column ( $300 \AA \AA, 1.7 \mu \mathrm{m}, 2.1 \mathrm{~mm} \times 100 \mathrm{~mm}$ ). Salts were removed with $3 \%$ Buffer B ( $0.1 \%$ formic acid in acetonitrile) at $0.2 \mathrm{~mL} /$ $\mathrm{min}$ for $3 \mathrm{~min}$ and proteins were eluted using a linear gradient of Buffer B from $3 \%$ to $80 \%$ in $4 \mathrm{~min}$ at $0.2 \mathrm{~mL} / \mathrm{min}$. The UPLC was coupled directly with a Synapt G2 HDMS qTOF mass spectrometer scanning $400-2500 \mathrm{~m} / z$. Charge deconvolution was performed using Waters MaxEnt software.

For acetylation sites analysis, the trypsinized peptides were resuspended in $0.1 \%$ TFA, 3\% acetonitrile in water, of which 1 picomole of the peptides for each sample was directly injected onto a Waters M-class column $(1.7 \mu \mathrm{m}, 120 \mathrm{~A}, \mathrm{rpC18}, 75$ $\mu \mathrm{m} \times 250 \mathrm{~mm}$ ) and gradient eluted from $2 \%$ to $40 \%$ acetonitrile over $40 \mathrm{~min}$ at 0.3 $\mu \mathrm{L} / \mathrm{min}$ using a Thermo Ultimate 3000 UPLC (Thermo Scientific). Peptides were detected with a Thermo Q-Exactive HF-X mass spectrometer (Thermo Scientific) scanning MS1 spectra at 120,000 resolution from 380 to $1580 \mathrm{~m} / z$ with a $45 \mathrm{~ms}$ fill time and 3E6 AGC target. The top 12 most intense peaks were isolated with a $1.4 \mathrm{~m} / \mathrm{z}$ window with a $100 \mathrm{~ms}$ fill time and 1E6 AGC target and 27\% HCD collision energy for MS2 spectra collected at 15,000 resolution. Dynamic exclusion was enabled for $5 \mathrm{~s}$. MS data raw files were searched against the single Uniprot sequence for EP300 (Uniprot accession number Q09472) using Maxquant 1.6.14.0 with cysteine carbamidomethylation as a fixed modification, while methionine oxidation and protein $\mathrm{N}$-terminal and lysine side chain acetylation were set as variable modifications. The mass tolerances for the database search were $4.5 \mathrm{ppm}$ for the precursors and $20 \mathrm{ppm}$ for the MS2 fragment ions, the minimum peptide length was 7 residues with no additional applied score cutoffs. Peptide and protein level FDR was set at 0.01 . An intensity cut-off of 50 million counts was applied to the untreated sample prior to plotting the change in intensity in Fig. 2 j.

In vitro condensate formation. All in vitro condensate formation assays were performed in a buffer containing $15 \mathrm{mM}$ Tris- $\mathrm{HCl}(\mathrm{pH} 7.5), 150 \mathrm{mM} \mathrm{NaCl}$ and 2 mM DTT unless otherwise stated. All samples were prepared on ice and incubated for $\sim 5$ min before imaging on siliconized glass cover slides (Hampton). For WT and mutant $\mathrm{p} 300_{\mathrm{BRPHZ}}$ and $\mathrm{p} 300_{\mathrm{HZ}}$ alone, $13 \mu \mathrm{M}$ protein samples were parallelly prepared with or without $12 \%(\mathrm{w} / \mathrm{v})$ PEG 3350 . For the condensate formation assay with reconstituted nucleosomes, $6.4 \mu \mathrm{M}$ SIRT2-treated $\mathrm{p} 300_{\mathrm{BRPHZ}}$ and an equal amount of NCP were incubated in a buffer containing $10 \mathrm{mM}$ Tris $7.5,60 \mathrm{mM}$ $\mathrm{NaCl}, 2 \mathrm{mM}$ DTT, and 12\% (w/v) PEG350. Microscopy of the droplets was done using an M150C-I microscope (AmScope) equipped with a $\times 10$ objective and an MD35 digital camera (AmScope). A microscope camera calibration slide (OMAX, $0.01 \mathrm{~mm}$ ) was used to determine the scale. A $50 \mu \mathrm{m} \times 50 \mu \mathrm{m}$ square area was selected as a representative image for each sample. The number of condensates was also counted in a $50 \mu \mathrm{m} \times 50 \mu \mathrm{m}$ square area. Five non-overlapping square regions were counted for each sample and plotted. Experiments were repeated in at least three batches of purified and SIRT2-treated p300 proteins.

To prepare p300 condensates that concentrate DNA or H3, $13 \mu \mathrm{M}$ SIRT2treated p300 was mixed with either $6 \mu \mathrm{M}$ FAM, $6 \mu \mathrm{M}$ FAM-labeled $37 \mathrm{bp}$ dsDNA, or $6 \mu \mathrm{M}$ FAM-labeled histone $\mathrm{H} 3$ tail (aa 1-12). Confocal images were acquired on a Zeiss Observer.Z1 inverted microscope using a $\times 40$ oil objective and digitally captured. For the excitation of FAM, a $488 \mathrm{~nm}$ laser was used. Images were processed and presented using ImageJ and Photoshop.

Nucleosome reconstitution. The poly-cistronic vector of Xenopus laevis Histone octamers was obtained from the Jean-Francois Couture lab. A His-tag and a TEV cleavage site were introduced before histone $\mathrm{H} 3$ by mutagenesis. Expression and purification of histone octamers were performed as reported previously ${ }^{53}$. The Histag before histone $\mathrm{H} 3$ was cleaved to expose the Alal residue using TEV protease 601 DNA was prepared as previously described ${ }^{54}$. NCPs were reconstituted by combining octamer with $1.1 \times$ excess DNA and performing slow salt dialysis. Reconstituted nucleosomes were further purified by size-exclusion chromatography and concentrated in Millipore concentrators.

Acetyltransferase assays. Purified untreated and SIRT2-treated p $300_{\mathrm{BRPHZ}}$ proteins were buffer exchanged into reaction buffer containing $10 \mathrm{mM}$ Tris $-\mathrm{HCl}$ (pH 7.5) and $60 \mathrm{mM} \mathrm{NaCl}$. For auto-acetylation experiments in Fig. 3a-c, $10 \mu \mathrm{M}$ p300 protein was incubated in HAT reaction buffer $(10 \mathrm{mM}$ Tris $\mathrm{pH} 7.5$ and 60 $\mathrm{mM} \mathrm{NaCl}$ ) in the absence or presence of $12 \%$ (w/v) PEG 3350. For histone acetyltransferase assays in Fig. $6 \mathrm{j}, 6.4 \mu \mathrm{M}$ p300 protein, and $6.4 \mu \mathrm{M}$ reconstituted NCP were incubated in HAT reaction buffer $(10 \mathrm{mM}$ Tris $\mathrm{pH} 7.5$ and $60 \mathrm{mM} \mathrm{NaCl})$. Reactions were started by adding $0.5 \mathrm{mM}$ acetyl-CoA at room temperature and quenched by flash-freeze at indicated time points. For each time point, $8 \mu \mathrm{l}$ reaction mixture was diluted to $200 \mu \mathrm{l}$ assay buffer and immediately heated to $95^{\circ} \mathrm{C}$ for 5 min to inactivate $\mathrm{p} 300$. The samples were then applied to an ultrafiltration device (10k cut-off) to collect flow-through for CoA quantification by the fluorometric assay kit (Abcam, 138889). The kit utilizes a fluorogenic green indicator that became strongly fluorescent upon reacting with the $-\mathrm{SH}$ group in CoA. The fluorescence signals in samples collected from the reactions were measured according to the product manual using a 96-well microplate reader. For measuring the HAT activity of p300 (Fig. 6g-i) in the diluted solution, droplet mixture, and supernatant, reactions were quenched by mixing with a denaturing buffer containing $20 \mathrm{mM}$ Tris- $\mathrm{HCl}$ (pH 7.5), $150 \mathrm{mM} \mathrm{NaCl}$ and $6 \mathrm{M}$ guanidine- $\mathrm{HCl}$ at indicated points. All experiments were performed in triplicates.

To detect histone $\mathrm{H} 3$ acetylation at specific lysine sites, reactions were quenched by flash-freezing in liquid nitrogen and then analyzed by SDS-PAGE and western blot analysis. Western blot results were quantified by LI-COR Odyssey System using the following antibodies: anti-H3K4ac (ab176799, 1:1000), anti-H3K9ac (ab4441, 1:1000), and anti-H3K27ac (ab177178, 1:1000) from Abcam, and antiH3K18ac (39755, 1:1000) from Active Motif.

SAXS analysis. X-ray scattering data were collected at the Bio-SAXS beamline (BM29) of the European Synchrotron Radiation Facility. Data were collected with 
photon-counting Pilatus $1 \mathrm{M}$ detector at a sample-detector distance of $2.86 \mathrm{~m}$, a wavelength of $\lambda=0.991 \AA$, and an exposure time of 1 second/frame. A momentum transfer range of $0.008-0.47 \AA^{-1}$ was covered $(q=4 \pi \sin \theta / \lambda$, where $\theta$ is the scattering angle and $\lambda$ the X-ray wavelength). A time-resolved SAXS experiment was performed with the p300 (aa 324-2094) and $\triangle$ AIL p300 constructs, produced as described $^{55}$, during auto-acetylation. $150 \mu \mathrm{L}$ of the reaction mixtures in buffer $(20$ mM HEPES pH7.0, $500 \mathrm{mM} \mathrm{NaCl}, 5 \mu \mathrm{M} \mathrm{ZnCl}_{2}, 0.5 \mathrm{mM}$ TCEP) were prepared and pipetted in the thermostated sample holder tube already adjusted to $30^{\circ} \mathrm{C} .2 \mathrm{mM}$ acetyl-CoA was added to each reaction tube and in the background, buffer to start the reaction. Four scattering curves were recorded on each sample at $0,30,60$, and $90 \mathrm{~min} . R_{\mathrm{g}}$ and $I(0)$ values were obtained from the Guinier approximation $R_{\mathrm{g}}<1.3$ using Primus ${ }^{56}$. Distance distribution functions $p(r)$ were computed from the entire scattering curve using $\mathrm{GNOM}^{56}$.

EMSA with DNA and nucleosomes. EMSA experiments were performed essentially as described ${ }^{57}$. Briefly, increasing amounts of p300 were incubated with $147 \mathrm{bp} 601$ DNA $(50 \mathrm{nM})$ in a DNA binding buffer containing $20 \mathrm{mM}$ Tris- $\mathrm{HCl}$ (pH 7.5), 50 $\mathrm{mM} \mathrm{NaCl}$, and $2 \mathrm{mM}$ DTT for $5 \mathrm{~min}$. The reaction mixtures were loaded on $5 \%$ polyacrylamide gels, and electrophoresis was performed in $0.2 \times \mathrm{TBE}$ buffer at $100 \mathrm{~V}$ for $1.5 \mathrm{~h}$ on ice. For EMSA experiments with reconstituted nucleosomes $(100 \mathrm{nM})$, the $\mathrm{NaCl}$ concentration in binding buffer was increased to $75 \mathrm{mM}$, and electrophoresis was performed in $0.2 \times \mathrm{TB}$ buffer. Gels were stained with SYBR Gold (Invitrogen).

ChIP-seq analysis. Flag-p $300_{\text {BRPHZT }}$, H3K18ac, and H3K27ac ChIP-seq reads were obtained from GSE109591; H3K4me3 and H3K27me3 ChIP-seq reads were from GSE81322. ChIP-seq raw reads were mapped to the hg38 genome by hisat2 (v2.1.0) with no-spliced-alignment, -k 1. H3K4me3 and H3K27me3 peaks were called by macs2 callpeak (v2.1.2) with broad parameter. Flag-p $300_{\text {BRPHZT }}$ peaks were converted to hg38 by UCSC liftOver. Heatmaps were generated by danpos (v2.2.2) with 200 bp bin size and visualized by TreeView (v1.1.6).

Immunofluorescence. HeLa cells were cultured in a six-well plate with 22-mm coverslips. YFP-p300 plasmids were transfected into HeLa cells by Lipofectamine 3000 (Life Technology, L3000-075) according to manufacturer instructions. One day after transfection, cells were fixed by $1 \%$ paraformaldehyde and then permeabilized with $0.2 \%$ Triton X-100. After blocking with $3 \%$ goat serum and $3 \%$ BSA, cells were incubated, respectively, with pairs of primary antibodies: anti-GFP (Life Technologies; A-11120; 1:400 dilution) and anti-H3K27me3 (Millipore; 07449; 1:200 dilution), anti-GFP (Life Technologies; A-11120; 1:400 dilution) and anti-H3K9me3 (Upstate; 07-442; 1:200 dilution), and anti-GFP (Life Technologies; A-11120; 1:400 dilution) and anti-H3K4me3 (Novus; NB21-1023B; 1:200 dilution), for two hours. After washing, cells were incubated with a pair of secondary antibodies: Alexa Fluor 488-labeled goat anti-mouse and Alexa Fluor 568-labeled goat anti-rabbit, for two hours, and then were mounted by using ProLong Antifade reagents (Life Technologies; P7481). Line plots were made using the plot profile feature of ImageJ. Pearson coefficient correlation for each pair of immunofluorescence images was calculated using the Coloc 2 plugin in ImageJ after the selection of the entire cell nuclei as the regions of specific interest.

\section{Data availability}

All relevant data supporting the key findings of this study are provided in the Supplementary Information and Source Data files or from the corresponding author upon reasonable request. The mass spec data generated in this study have been deposited to the PRIDE database under the accession number PXD026898. The publicly available ChIP-seq data analyzed in this study are available from Gene Expression Omnibus under accession codes GSE109591 and GSE81322. Source data are provided with this paper.

Received: 8 March 2021; Accepted: 12 July 2021;

Published online: 29 July 2021

\section{References}

1. Shin, Y. et al. Liquid nuclear condensates mechanically sense and restructure the genome. Cell 175, 1481-1491 (2018). e1413.

2. Brangwynne, C. P., Mitchison, T. J. \& Hyman, A. A. Active liquid-like behavior of nucleoli determines their size and shape in Xenopus laevis oocytes. Proc. Natl Acad. Sci. USA 108, 4334-4339 (2011).

3. Li, P. et al. Phase transitions in the assembly of multivalent signalling proteins. Nature 483, 336-340 (2012).

4. Banani, S. F., Lee, H. O., Hyman, A. A. \& Rosen, M. K. Biomolecular condensates: organizers of cellular biochemistry. Nat. Rev. Mol. Cell Biol. 18 , 285-298 (2017).

5. Gibson, B. A. et al. Organization of chromatin by intrinsic and regulated phase separation. Cell 179, 470-484 (2019). e421.
6. Sabari, B. R. et al. Coactivator condensation at super-enhancers links phase separation and gene control. Science 361, eaar3958 (2018).

7. Larson, A. G. et al. Liquid droplet formation by HPlalpha suggests a role for phase separation in heterochromatin. Nature 547, 236-240 (2017).

8. Strom, A. R. et al. Phase separation drives heterochromatin domain formation. Nature 547, 241-245 (2017).

9. Hnisz, D., Shrinivas, K., Young, R. A., Chakraborty, A. K. \& Sharp, P. A. A phase separation model for transcriptional control. Cell 169, 13-23 (2017).

10. Boija, A. et al. Transcription factors activate genes through the phaseseparation capacity of their activation domains. Cell 175, 1842-1855e1816 (2018).

11. Zhang, Y. et al. MORC3 forms nuclear condensates through phase separation. iScience 17, 182-189 (2019).

12. Plys, A. J. et al. Phase separation of Polycomb-repressive complex 1 is governed by a charged disordered region of CBX2. Genes Dev. 33, 799-813 (2019).

13. Cho, W. K. et al. Mediator and RNA polymerase II clusters associate in transcription-dependent condensates. Science 361, 412-415 (2018).

14. Nair, S. J. et al. Phase separation of ligand-activated enhancers licenses cooperative chromosomal enhancer assembly. Nat. Struct. Mol. Biol. 26, 193-203 (2019).

15. Boehning, M. et al. RNA polymerase II clustering through carboxy-terminal domain phase separation. Nat. Struct. Mol. Biol. 25, 833-840 (2018).

16. Tatavosian, R. et al. Nuclear condensates of the Polycomb protein chromobox 2 (CBX2) assemble through phase separation. J. Biol. Chem. 294, 1451-1463 (2019).

17. Liu, X. et al. The structural basis of protein acetylation by the $\mathrm{p} 300 / \mathrm{CBP}$ transcriptional coactivator. Nature 451, 846-850 (2008).

18. Ogryzko, V. V., Schiltz, R. L., Russanova, V., Howard, B. H. \& Nakatani, Y. The transcriptional coactivators p300 and CBP are histone acetyltransferases. Cell 87, 953-959 (1996).

19. Wang, Z. et al. Genome-wide mapping of HATs and HDACs reveals distinct functions in active and inactive genes. Cell 138, 1019-1031 (2009).

20. Dancy, B. M. \& Cole, P. A. Protein lysine acetylation by p300/CBP. Chem. Rev 115, 2419-2452 (2015).

21. Goodman, R. H. \& Smolik, S. CBP/p300 in cell growth, transformation, and development. Genes Dev. 14, 1553-1577 (2000).

22. Jin, Q. et al. Distinct roles of GCN5/PCAF-mediated H3K9ac and CBP/p300mediated $\mathrm{H} 3 \mathrm{~K} 18 / 27 \mathrm{ac}$ in nuclear receptor transactivation. EMBO J. 30, 249-262 (2011).

23. Tang, Z. et al. SET1 and p300 act synergistically, through coupled histone modifications, in transcriptional activation by p53. Cell 154, 297-310 (2013)

24. Gu, W. \& Roeder, R. G. Activation of p53 sequence-specific DNA binding by acetylation of the p53 C-terminal domain. Cell 90, 595-606 (1997).

25. Zhang, Y. et al. The ZZ domain of p300 mediates specificity of the adjacent HAT domain for histone H3. Nat. Struct. Mol. Biol. 25, 841-849 (2018).

26. Thompson, P. R. et al. Regulation of the p300 HAT domain via a novel activation loop. Nat. Struct. Mol. Biol. 11, 308-315 (2004).

27. Delvecchio, M., Gaucher, J., Aguilar-Gurrieri, C., Ortega, E. \& Panne, D. Structure of the p300 catalytic core and implications for chromatin targeting and HAT regulation. Nat. Struct. Mol. Biol. 20, 1040-1046 (2013).

28. Ortega, E. et al. Transcription factor dimerization activates the p300 acetyltransferase. Nature 562, 538-544 (2018).

29. Rada-Iglesias, A. et al. A unique chromatin signature uncovers early developmental enhancers in humans. Nature 470, 279-283 (2011).

30. Zentner, G. E., Tesar, P. J. \& Scacheri, P. C. Epigenetic signatures distinguish multiple classes of enhancers with distinct cellular functions. Genome Res. 21, 1273-1283 (2011)

31. Holmqvist, P. H. \& Mannervik, M. Genomic occupancy of the transcriptional co-activators p300 and CBP. Transcription 4, 18-23 (2013).

32. Bedford, D. C., Kasper, L. H., Fukuyama, T. \& Brindle, P. K. Target gene context influences the transcriptional requirement for the KAT3 family of CBP and p300 histone acetyltransferases. Epigenetics 5, 9-15 (2010).

33. Kasper, L. H., Qu, C., Obenauer, J. C., McGoldrick, D. J. \& Brindle, P. K. Genome-wide and single-cell analyses reveal a context dependent relationship between CBP recruitment and gene expression. Nucleic Acids Res. 42, 11363-11382 (2014)

34. Girdwood, D. et al. P300 transcriptional repression is mediated by SUMO modification. Mol. Cell 11, 1043-1054 (2003).

35. Boisvert, F. M., Kruhlak, M. J., Box, A. K., Hendzel, M. J. \& Bazett-Jones, D. P The transcription coactivator CBP is a dynamic component of the promyelocytic leukemia nuclear body. J. Cell Biol. 152, 1099-1106 (2001).

36. LaMorte, V. J., Dyck, J. A., Ochs, R. L. \& Evans, R. M. Localization of nascent RNA and CREB binding protein with the PML-containing nuclear body. Proc Natl Acad. Sci. USA 95, 4991-4996 (1998).

37. McManus, K. J. \& Hendzel, M. J. Quantitative analysis of CBP- and P300induced histone acetylations in vivo using native chromatin. Mol. Cell. Biol. 23, 7611-7627 (2003) 
38. Sabari, B. R. et al. Intracellular crotonyl-CoA stimulates transcription through p300-catalyzed histone crotonylation. Mol. Cell 58, 203-215 (2015).

39. Wang, Y. et al. p300 Acetyltransferase is a cytoplasm-to-nucleus shuttle for SMAD2/3 and TAZ nuclear transport in transforming growth factor betastimulated hepatic stellate cells. Hepatology 70, 1409-1423 (2019).

40. Bandobashi, K. et al. Intranuclear localization of the transcription coadaptor $\mathrm{CBP} / \mathrm{p} 300$ and the transcription factor RBP-Jk in relation to EBNA-2 and -5 in B lymphocytes. Virology 288, 275-282 (2001).

41. Karanam, B., Jiang, L., Wang, L., Kelleher, N. L. \& Cole, P. A. Kinetic and mass spectrometric analysis of $\mathrm{p} 300$ histone acetyltransferase domain autoacetylation. J. Biol. Chem. 281, 40292-40301 (2006).

42. Black, J. C., Mosley, A., Kitada, T., Washburn, M. \& Carey, M. The SIRT2 deacetylase regulates autoacetylation of p300. Mol. cell 32, 449-455 (2008).

43. Park, S. et al. Role of the CBP catalytic core in intramolecular SUMOylation and control of histone H3 acetylation. Proc. Natl Acad. Sci. USA 114, E5335-E5342 (2017).

44. Zhang, Y., Narlikar, G. J. \& Kutateladze, T. G. Enzymatic reactions inside biological condensates. J. Mol. Biol. 433, 166624, (2021).

45. Prouteau, M. et al. TORC1 organized in inhibited domains (TOROIDs) regulate TORC1 activity. Nature 550, 265-269 (2017).

46. Drobot, B. et al. Compartmentalised RNA catalysis in membrane-free coacervate protocells. Nat. Commun. 9, 3643 (2018).

47. Schutz, S., Noldeke, E. R. \& Sprangers, R. A synergistic network of interactions promotes the formation of in vitro processing bodies and protects mRNA against decapping. Nucleic Acids Res. 45, 6911-6922 (2017).

48. Buecker, C. \& Wysocka, J. Enhancers as information integration hubs in development: lessons from genomics. Trends Genet. 28, 276-284 (2012).

49. Holmqvist, P. H. et al. Preferential genome targeting of the CBP co-activator by Rel and Smad proteins in early Drosophila melanogaster embryos. PLoS Genet. 8, e1002769 (2012).

50. Chen, J. \& Li, Q. Life and death of transcriptional co-activator p300. Epigenetics 6, 957-961 (2011).

51. Chong, S. et al. Imaging dynamic and selective low-complexity domain interactions that control gene transcription. Science 361, eaar2555 (2018).

52. Hughes, C. S. et al. Ultrasensitive proteome analysis using paramagnetic bead technology. Mol. Syst. Biol. 10, 757 (2014).

53. Shim, Y., Duan, M. R., Chen, X., Smerdon, M. J. \& Min, J. H. Polycistronic coexpression and nondenaturing purification of histone octamers. Anal. Biochem. 427, 190-192 (2012).

54. Klein, B. J. et al. Histone H3K23-specific acetylation by MORF is coupled to H3K14 acylation. Nat. Commun. 10, 4724 (2019).

55. Kaczmarska, Z. et al. Structure of p300 in complex with acyl-CoA variants. Nat. Chem. Biol. 13, 21-29 (2017).

56. Petoukhov, M. V. et al. New developments in the ATSAS program package for small-angle scattering data analysis. J. Appl. Crystallogr. 45, 342-350 (2012).

57. Klein, B. J. et al. Bivalent interaction of the PZP domain of BRPF1 with the nucleosome impacts chromatin dynamics and acetylation. Nucleic Acids Res. 44, 472-484 (2016).

\section{Acknowledgements}

We thank Dr. Berk for providing cDNA of YFP-p300. The authors thank Cole Michel at the University of Colorado School of Pharmacy Mass Spectrometry Facility for help with collecting and analyzing samples. This work was supported by grants from NIH GM125195, GM135671, HL151334, CA252707, and AG067664 to T.G.K., R01GM135286 to X.R. and CA204020 to X.S. Y.Z. is supported by NIH K99CA241301.

\section{Author contributions}

Y.Z., K.B., Y.Y., Z.I., M.Z., H.X., S.I., T.L., C.C.E., and J.L. performed experiments and together with D.P., X.S., X.R., and T.G.K. analyzed the data. Y.Z. and T.G.K. wrote the manuscript with input from all authors.

\section{Competing interests}

The authors declare no competing interests.

\section{Additional information}

Supplementary information The online version contains supplementary material available at https://doi.org/10.1038/s41467-021-24950-8.

Correspondence and requests for materials should be addressed to T.G.K.

Peer review information Nature Communications thanks Dong Wang and the other, anonymous, reviewers for their contribution to the peer review of this work. Peer reviewer reports are available.

Reprints and permission information is available at http://www.nature.com/reprints Publisher's note Springer Nature remains neutral with regard to jurisdictional claims in published maps and institutional affiliations.

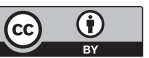

Open Access This article is licensed under a Creative Commons Attribution 4.0 International License, which permits use, sharing, adaptation, distribution and reproduction in any medium or format, as long as you give appropriate credit to the original author(s) and the source, provide a link to the Creative Commons license, and indicate if changes were made. The images or other third party material in this article are included in the article's Creative Commons license, unless indicated otherwise in a credit line to the material. If material is not included in the article's Creative Commons license and your intended use is not permitted by statutory regulation or exceeds the permitted use, you will need to obtain permission directly from the copyright holder. To view a copy of this license, visit http://creativecommons.org/ licenses/by/4.0/

(c) The Author(s) 2021 